\title{
Surface-Tension- and Injection-Driven Spreading of a Thin Viscous Film
}

\author{
K. B. Kiradjiev ${ }^{1} \dagger$, C. J. W. Breward ${ }^{1}$ and I. M. Griffiths ${ }^{1}$ \\ ${ }^{1}$ Mathematical Institute, University of Oxford, Radcliffe Observatory Quarter, Woodstock Rd, \\ Oxford OX2 6GG
}

(Received $\mathrm{xx}$; revised $\mathrm{xx}$; accepted $\mathrm{xx}$ )

We consider the spreading of a thin viscous droplet, injected through a finite region of a substrate, under the influence of surface tension. We neglect gravity and assume that there is a precursor layer covering the whole substrate and that the rate of injection is constant. We analyse the evolution of the film profile for early and late time, and obtain power-law dependencies for the maximum film thickness at the centre of the injection region and the position of an apparent contact line, which compare well with numerical solutions of the full problem. We relax the conditions on the injection rate to consider more general time-dependent and spatially varying forms. In the case of power-law injection of the form $t^{k}$, we observe a switch in the behaviour of the evolution of the film thickness for late time from increasing to decreasing at a critical value of $k$. We show that point-source injection can be treated as a limiting case of a finiteinjection slot and the solutions exhibit identical behaviours for late time. Finally, we formulate the problem with thickness-dependent injection rate, discuss the behaviour of the maximum film thickness and the position of the apparent contact line, and give power-law dependencies for these.

\section{Key words:}

\section{Introduction}

Surface-tension-driven thin-film flows arise in a wide range of physical applications. For example, they are crucial in understanding the spreading of liquid films (Oron et al. 1997; Craster \& Matar 2009; Zheng et al. 2018), drying of paints (Howison et al. 1997), biological processes such as coating of the internal sides of the airways (Halpern \& Grotberg 1992), contact-lens manufacture (Murphy \& Lee 2017), a vast variety of coating processes in industry (Wilson 1982; Howell et al. 2013) including spin-coating (Fraysse \& Homsy 1994; Wilson et al. 2000), and spreading of chemical reaction fronts in liquids (Guzman \& Vasquez 2016). While the spreading of a fixed volume of fluid is well-studied (see, e.g., Voinov (1976), Hocking (1980), Lacey (1982), Hocking (1983), Cox (1986), Ehrhard \& Davis (1991), Hocking (1992), King \& Bowen (2001), Bonn et al. (2009), Savva \& Kalliadasis (2009), Howell (2010), Ajaev (2012), Savva \& Kalliadasis (2013)), the analysis of thin-film flows with sources and sinks is much less common. These flows arise in numerous interesting applications, such as oozing of lava from a volcanic crater (Schwartz \& Michaelides 1988), spreading of films on a porous bed such as textile fabric (Davis \& Hocking 1999, 2000; Mason \& Momoniat 2004; Thompson

$\dagger$ Email address for correspondence: kristian.kiradjiev@maths.ox.ac.uk 
et al. 2015), and droplet spreading with evaporation (Anderson \& Davis 1995; Oliver et al. 2015; Saxton et al. 2016). Our interest in thin-film flows with injection is motivated by studying the operation of a purification device that removes gaseous chemicals from an airstream by converting them to liquid through a surface reaction in part of the device. Such injection-spreading problems have been studied in the case when gravity is the driving force for thin-film source flow on an inclined plate (Smith 1973; Huppert 1982; Schwartz \& Michaelides 1988; Lister 1992), and numerical and similarity solutions have been obtained for the resulting film thickness profile. In particular, Huppert (1982) also considers the case of a time-dependent source. Duffy \& Moffatt (1997) study a three-dimensional gravity-driven source flow down an almost vertical plane with surfacetension effects included in the steady-flow regime. They find power-law dependencies on the distance down the plane for the width and the height of the resulting film. Further analysis of gravity-driven rivulet flow of Newtonian and power-law fluids down an inclined plane is given in Yatim et al. (2010), whereas shear-stress-driven flow is considered in Yatim et al. (2012). Corresponding travelling-wave similarity solutions are found in Yatim et al. (2013). Momoniat et al. (2010) consider a similar problem but for radially symmetric spreading with an annular injection slot and investigate the flow behaviour numerically. In Mason \& Momoniat (2004) and Momoniat \& Mason (2007), symmetry methods from Lie algebras are used to study thin-film flow with injection/suction acting over a porous bed. The related problem of the flow of a viscous film with injection on an inclined porous plane is studied by Thompson et al. (2015). Here, both surface tension and gravity are included, but the spatial variations of the source term are assumed to be periodic and have zero mean, so that steady-state solutions can be analysed. In addition, the linear stability of these steady states is considered, which reveals that suction and blowing can either increase or decrease the critical Reynolds number for linear stability of these steady states. A different problem is studied in Hocking et al. (2011), where the blowing is present due to an air jet directed towards a thin film of steel, with the goal of facilitating the coating process. Similar air-jet blowing has been studied in Chilukuri et al. (1984) and McKinley et al. (1999), for example. Problems involving thin-film flow with injection of liquid are also studied in the context of elasticity. A concise treatment of the problem of an elastic sheet over a continuously injected viscous fluid is presented in Lister et al. (2013). Here, the process under consideration is the peeling of the sheet by bending and pulling at the front facilitated by the injected liquid, and they derive specific propagation laws (governed by the effect of elasticity of the covering membrane) with power-law dependence on time. They also assume the viscous film is situated over a thin pre-wetted layer. A similar problem is considered in Zheng et al. (2015), where buoyancy-driven spreading of a thin film over a membrane with constant injection of liquid is studied. Neglecting the effect of surface tension, they obtain similarity solutions for the position of the liquid front for late time.

A comprehensive review of thin-film equations and spreading is given in Myers (1998), where various thin-film equations are derived. There is a well-known stress singularity at the moving contact line, which is often resolved by assuming that there is precursor layer that pre-wets the substrate and removes the singularity. Such precursor layers are also observed in many physical systems (Bonn et al. 2009; Ajaev 2012). A comparison between different regularizations (in particular, slip and precursor regularizations) is presented in Savva \& Kalliadasis (2011) and Sibley et al. (2015). The authors conclude that, for certain parameter regimes, the regularizations studied lead to equivalent dropletspreading behaviours. Asymptotic results for the depth and extent of a steady thin-film flow down an inclined plane that is pre-wetted with a thin precursor layer are given in Benilov et al. (2010). In our work, we will be guided by the results obtained in King \& 
Bowen (2001), in which they analyse a general mass-preserving surface-tension-dominated lubrication equation with a very thin precursor layer. However, their results only hold for the spreading of a fixed volume of fluid and do not account for injection of liquid. Lacey (1982) takes a different approach by assuming a slip regularization, and derives asymptotic results for the spreading of a viscous droplet due to surface tension for late time. Anderson \& Davis (1995) consider steady and unsteady spreading of an evaporating droplet on a heated surface. They find a relationship between the contact angle and the rate of evaporation, and use asymptotic analysis in the large-capillary-number regime to determine the shape of the droplet. Oliver et al. (2015) consider the surface-tension-driven spreading of a partially wetting drop, assuming a slip regularization, with mass transfer along the entire free surface of the resulting film and, in particular, at the contact line. This leads to appropriate generalizations of Tanner's law. Similar results are obtained in Saxton et al. (2016), where the effect of evaporation on the evolution of the contact-set radius is studied. In Saxton et al. (2017), vapour transport and kinetic effects are used to regularize the mass-flux singularity at the contact line in an evaporating spreading droplet.

In this paper, we will study the surface-tension-driven flow of liquid from a finitewidth injection region over a precursor layer. We will explore the problem in which the precursor thickness tends to zero. Initially, we will consider the situation where the production rate is constant, and, therefore, the possibility of a steady state is ruled out. In $\S 2$, we formulate our model, explain and employ the precursor-layer regularization and then non-dimensionalize. In $\S 3$, we present our asymptotic analysis of the problem for early and late time, and briefly consider the $O(1)$-time problem. We will look for power-law predictions for the film thickness in the centre of the injection region, where it attains its maximum, and for the position of the apparent contact line in these temporal regimes. We will present numerical solutions to the full problem and compare them with the asymptotic predictions in $\S 4$. Finally, in $\S 5$ and $\S 6$, we will consider generalizations to our problem, in which we take more general time- and thickness-dependent source functions, respectively. Further, for power-law-in-time injection, we will suggest a way of predicting the power-law exponent by examining the film thickness profile and the position of the apparent contact line. We draw conclusions and discuss future work in $\S 7$.

\section{Mathematical Model}

\subsection{Formulation of the Dimensional Problem}

We consider an infinite one-dimensional substrate, on which viscous liquid is injected uniformly through the surface in the region $|\hat{x}| \leqslant L$ at a constant speed $W$. We let $\hat{x}$ measure the distance along the substrate and $\hat{z}$ measure the distance perpendicular to the substrate, and we show a schematic of the geometry under consideration in figure 1 . We expect the reduced Reynolds number to be very small for flows of practical interest in purification devices, and so, we ignore the effect of inertia. Further, assuming that gravity can also be neglected, the flow is surface-tension driven and governed by Stokes' equations

$$
\begin{aligned}
-\nabla \hat{p}+\mu \nabla^{2} \hat{\boldsymbol{u}} & =0, \\
\nabla \cdot \hat{\boldsymbol{u}} & =0
\end{aligned}
$$




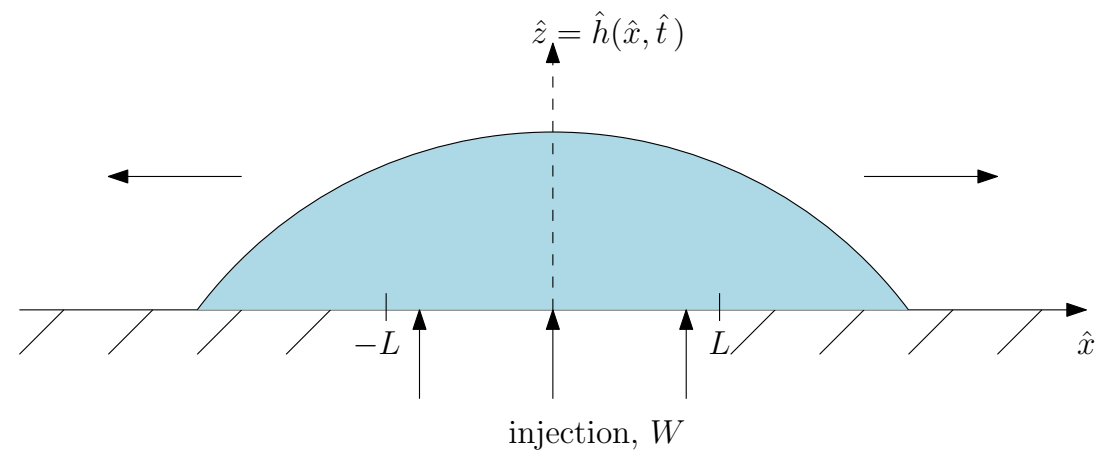

Figure 1: Schematic diagram of the thin-film flow problem with injection in the region $-L<\hat{x}<L$.

where $\hat{\boldsymbol{u}}=(\hat{u}, \hat{w})$ is the fluid velocity, $\hat{p}$ is the pressure, and $\mu$ is the viscosity of the liquid. We have the following no-slip, kinematic and stress boundary conditions,

$$
\begin{aligned}
& \hat{u}=0 \quad \text { on } \quad \hat{z}=0, \\
& \hat{w}=W \Theta(L-|\hat{x}|) \quad \text { on } \quad \hat{z}=0 \text {, } \\
& \hat{w}=\frac{\partial \hat{h}}{\partial \hat{t}}+\hat{u} \frac{\partial \hat{h}}{\partial \hat{x}} \quad \text { on } \quad \hat{z}=\hat{h}(\hat{x}, \hat{t}), \\
& \hat{\boldsymbol{n}} \cdot \hat{\boldsymbol{T}} \cdot \hat{\boldsymbol{n}}=\gamma(-\nabla \cdot \hat{\boldsymbol{n}})=\gamma \frac{\partial^{2} \hat{h}}{\partial \hat{x}^{2}}\left(1+\left(\frac{\partial \hat{h}}{\partial \hat{x}}\right)^{2}\right)^{-3 / 2} \quad \text { on } \quad \hat{z}=\hat{h}(\hat{x}, \hat{t}), \\
& \hat{\boldsymbol{n}} \cdot \hat{\boldsymbol{T}} \cdot \hat{\boldsymbol{t}}=0 \quad \text { on } \quad \hat{z}=\hat{h}(\hat{x}, \hat{t}),
\end{aligned}
$$

where $\Theta(\hat{x})$ is the Heaviside step function, $\hat{\boldsymbol{t}}$ and $\hat{\boldsymbol{n}}$ are the unit tangent and outwardpointing normal vectors to the free surface $\hat{z}=\hat{h}(\hat{x}, \hat{t})$, defined by

$$
\hat{\boldsymbol{t}}=\left(1+\left(\frac{\partial \hat{h}}{\partial \hat{x}}\right)^{2}\right)^{-1 / 2}\left(1, \frac{\partial \hat{h}}{\partial \hat{x}}\right), \quad \hat{\boldsymbol{n}}=\left(1+\left(\frac{\partial \hat{h}}{\partial \hat{x}}\right)^{2}\right)^{-1 / 2}\left(-\frac{\partial \hat{h}}{\partial \hat{x}}, 1\right)
$$

$\gamma$ is the (constant) surface tension, and $\hat{\boldsymbol{T}}$ is the stress tensor, defined by

$$
\hat{\boldsymbol{T}}=\left(\begin{array}{cc}
-\hat{p}+2 \mu \frac{\partial \hat{u}}{\partial \hat{x}} & \mu\left(\frac{\partial \hat{u}}{\partial \hat{z}}+\frac{\partial \hat{w}}{\partial \hat{x}}\right) \\
\mu\left(\frac{\partial \hat{u}}{\partial \hat{z}}+\frac{\partial \hat{w}}{\partial \hat{x}}\right) & -\hat{p}+2 \mu \frac{\partial \hat{w}}{\partial \hat{z}}
\end{array}\right)
$$

and evaluated on the free surface.

At the moving front, $\hat{x}=\hat{a}(\hat{t})$, say, we could impose zero-thickness and no-flux conditions along with a slip law along the whole of the substrate. For late time, as in the non-injection case we expect a quasi-static profile for the film thickness, where the relationship between the dynamic contact angle, $\theta_{d}$, and the speed of the contact line, $\dot{\hat{a}}$ (where a dot ( ) denotes differentiation with respect to time), is given by a version of Tanner's law. For small contact angles, this can be written as

$$
\frac{\partial \hat{h}}{\partial \hat{x}}=\hat{k} \dot{\hat{a}}^{1 / 3}
$$




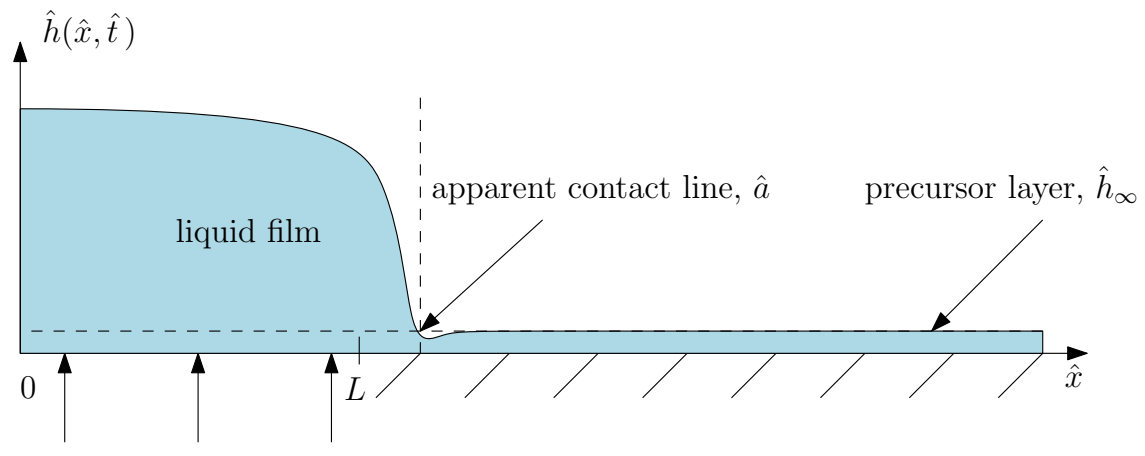

injection, $W$

Figure 2: A schematic diagram of the thin-film problem on half-space with injection and a precursor layer of thickness $\hat{h}_{\infty}$.

for some constant of proportionality $\hat{k}$, depending on the system under consideration (Tanner 1979; Myers 1998).

However, instead we choose to regularize the problem using a precursor-film regularization. In this regularization, we assume that the whole substrate is initially covered in a thin layer of liquid of thickness $\hat{h}_{\infty}$ (see figure 2 ). Thus, we assume that

$$
\hat{h} \rightarrow \hat{h}_{\infty} \quad \text { as } \quad \hat{x} \rightarrow \infty,
$$

and

$$
\hat{h}=\hat{h}_{\infty} \quad \text { at } \quad \hat{t}=0 .
$$

This regularization is sufficient to close the problem (King \& Bowen 2001), it resolves the well-known stress singularity that occurs at the moving contact line in film-spreading problems (Huh \& Scriven 1971), and we will find that this recovers a version of Tanner's law describing the motion of the apparent contact line, $\hat{a}$. The position of the apparent contact line, which lies in a narrow region where the film thickness adjusts to the precursor thickness, is sometimes defined as the point where the curvature of the free surface attains a local maximum (see, for example, Carlson et al. 2015; Ajaev 2012). However, any point in the narrow transition region can serve as a definition for the contact line. For concreteness, in this analysis, as well as the numerical solutions, we define the position of the contact line to be the smallest value of $\hat{x}$ at which $\hat{h}$ equals the precursor layer thickness, $\hat{h}_{\infty}$ (see figure 2 ).

Our final lateral boundary conditions are to assume symmetry at $\hat{x}=0$, i.e.,

$$
\hat{u}=\frac{\partial \hat{p}}{\partial \hat{x}}=\frac{\partial \hat{h}}{\partial \hat{x}}=\frac{\partial^{3} \hat{h}}{\partial \hat{x}^{3}}=0 \quad \text { at } \quad \hat{x}=0 .
$$

From now on, we assume $x \geqslant 0$ and will drop the modulus sign on the right-hand side of $(2.4)$.

\subsection{Dimensionless Model}

We non-dimensionalize (2.1)-(2.7) using

$$
\begin{gathered}
(\hat{x}, \hat{a})=L(x, a), \quad(\hat{z}, \hat{h})=\left(\mu W L^{4} / \gamma\right)^{1 / 4}(z, h), \\
\hat{u}=\left(\gamma W^{3} / \mu\right)^{1 / 4} u, \quad \hat{w}=W w, \quad \hat{t}=\left(\mu L^{4} / \gamma W^{3}\right)^{1 / 4} t, \quad \hat{p}=\left(\mu W \gamma^{3} / L^{4}\right)^{1 / 4} p .
\end{gathered}
$$


We note that we have chosen the intrinsic scale for $\hat{h}$ that emerges as a result of nondimensionalizing (2.6) and balancing viscous forces in the normal direction with surfacetension forces. We assume that the aspect ratio in the problem $\epsilon=(\mu W / \gamma)^{1 / 4} \ll 1$, and expand the dependent variables in asymptotic series in powers of $\epsilon^{2}$. This gives the following leading-order dimensionless problem

$$
\begin{aligned}
-\frac{\partial p}{\partial x}+\frac{\partial^{2} u}{\partial z^{2}} & =0 \\
-\frac{\partial p}{\partial z} & =0 \\
\frac{\partial u}{\partial x}+\frac{\partial w}{\partial z} & =0
\end{aligned}
$$

together with

$$
\begin{array}{rlrl}
u=0 & \text { on } & z=0, \\
w=\Theta(L-x) & \text { on } & z=0, \\
w=\frac{\partial h}{\partial t}+u \frac{\partial h}{\partial x} & \text { on } & z & =h(x, t), \\
p=-\frac{\partial^{2} h}{\partial x^{2}} & \text { on } & z & =h(x, t), \\
\frac{\partial u}{\partial z}=0 & \text { on } & z & =h(x, t) .
\end{array}
$$

Integrating (2.17), and using (2.15)-(2.16) together with (2.18)-(2.22), we find that $h$ satisfies the lubrication equation

$$
\frac{\partial h}{\partial t}+\frac{1}{3} \frac{\partial}{\partial x}\left(h^{3} \frac{\partial^{3} h}{\partial x^{3}}\right)=\Theta(1-x) .
$$

The dimensionless boundary and initial conditions become

$$
\begin{aligned}
& \frac{\partial h}{\partial x}=\frac{\partial^{3} h}{\partial x^{3}}=0 \quad \text { at } \quad x=0, \\
& h \rightarrow \delta \quad \text { as } \quad x \rightarrow \infty, \\
& h=\delta \quad \text { at } \quad t=0 \text {, }
\end{aligned}
$$

where $\delta=\hat{h}_{\infty} /(\epsilon L) \ll 1$ is the dimensionless precursor film thickness and the only remaining parameter in the system. Although we have only three boundary conditions for our fourth-order equation, the precursor-layer regularization (2.25) is enough to ensure a unique solution (King \& Bowen 2001; Myers 1998).

Integrating (2.23) with respect to both $x$ and $t$ and applying (2.24) and (2.26), we obtain

$$
\int_{0}^{\infty}(h(x, t)-\delta) \mathrm{d} x=t
$$

Equation (2.27) is an alternative expression for global conservation of mass, but will be convenient to use in the following analysis.

In the next section, we will explore the behaviour of the film thickness for early, $O(1)$, and late time, which will enable us to make substantial analytical progress with the problem. 


\section{Asymptotic Analysis in the Limit $\delta \ll 1$}

We begin by considering the asymptotic behaviour in $\delta$ of (2.23)-(2.27) for early, $O(1)$, and late time, and will later compare the results with the full numerical solution. We will find that (i) it is straightforward to determine the early-time behaviour, (ii) the $O(1)$-time behaviour is identical to the case of zero injection, and (iii) the interesting injection-dependent behaviour occurs at late time.

\subsection{Early-Time Behaviour}

To examine the early-time behaviour of $h$, we rescale the thickness $h$ with the initial thickness, $\delta$, and scale time to balance the film growth with the liquid supply, i.e., we set

$$
h=\delta \tilde{h}, \quad t=\delta \tilde{t} .
$$

We pose the asymptotic expansions

$$
\begin{aligned}
\tilde{h}(x, \tilde{t}) \sim \tilde{h}_{0}(x, \tilde{t})+\delta \tilde{h}_{1}(x, \tilde{t})+\cdots & \text { as } & \delta \rightarrow 0, \\
a(\tilde{t}) \sim 1+\delta a_{1}(\tilde{t})+\cdots & \text { as } \quad & \delta \rightarrow 0,
\end{aligned}
$$

and assume, following King \& Bowen (2001), that on this timescale the apparent contact line moves only an $O(\delta)$ distance from its original location at the end of the injection region. Thus, to leading order we have

$$
\frac{\partial \tilde{h}_{0}}{\partial \tilde{t}}=\Theta(1-x)
$$

with initial condition

$$
\tilde{h}_{0}=1 \quad \text { at } \quad \tilde{t}=0 .
$$

The solution, in terms of the original variables, is

$$
h_{0}(x, t)=\delta+t \Theta(1-x) .
$$

We immediately note that, since this solution is spatially uniform on either side of $x=1$, the boundary conditions (2.24) are automatically satisfied. The global conservation of mass (2.27) also holds. However, there is a discontinuity in our solution at $x=1$ and, to determine the smooth transition between the two solutions given in (3.6), we need to consider a boundary layer near $x=1$. Specifically, we rescale

$$
x=1+\delta \tilde{x} .
$$

In this boundary layer, we obtain the following problem

$$
\frac{\partial \tilde{h}}{\partial \tilde{t}}+\frac{1}{3} \frac{\partial}{\partial \tilde{x}}\left(\tilde{h}^{3} \frac{\partial^{3} \tilde{h}}{\partial \tilde{x}^{3}}\right)=\Theta(-\tilde{x}),
$$

subject to

$$
\begin{array}{rll}
\tilde{h} \rightarrow 1 & \text { as } & \tilde{x} \rightarrow \infty, \\
\tilde{h} \rightarrow 1+\tilde{t} & \text { as } & \tilde{x} \rightarrow-\infty, \\
\tilde{h}=1 & \text { at } & \tilde{t}=0 .
\end{array}
$$

We will solve this boundary-layer problem numerically in $\S 4$.

Before we focus on the behaviour of the thickness of the liquid film $h(x, t)$ for late time when we expect $O(1)$ movement of the contact line, we look at the $O(1)$-time problem and discuss the behaviour of the apparent contact line. 


\subsection{O(1)-Time Behaviour}

For $O(1)$ time, all terms in (2.23) come into play, and analytical progress is difficult. However, we will carefully analyse the motion of the apparent contact line with the purpose of exploiting these results during the late-time analysis. The scalings that we obtain in this section are identical to those used in the no-injection case considered in King \& Bowen (2001). They find that the apparent contact line is stationary for $O(1)$ time, which is supported by our numerical solutions found in $\S 4$. We will focus on the next-order correction to this leading-order behaviour. Following the work of King \& Bowen (2001), we note that the leading-order (in $\delta$ ) solution for $h$ in the injection region has the following behaviour as $x \rightarrow 1^{-}$

$$
h_{0} \sim A(t)(1-x) \quad \text { as } \quad x \rightarrow 1^{-},
$$

where $A(t)>0$ is some function that can, in theory, be determined from solving the leading-order problem

$$
\frac{\partial h_{0}}{\partial t}+\frac{1}{3} \frac{\partial}{\partial x}\left(h_{0}^{3} \frac{\partial^{3} h_{0}}{\partial x^{3}}\right)=1
$$

together with

$$
\begin{aligned}
& \frac{\partial h_{0}}{\partial x}=\frac{\partial^{3} h_{0}}{\partial x^{3}}=0 \quad \text { at } \quad x=0, \\
& h_{0}=0 \quad \text { at } \quad x=1 \text {, } \\
& h_{0}=0 \quad \text { at } \quad t=0 \text {, }
\end{aligned}
$$

and conservation of mass

$$
\int_{0}^{1} h_{0}(x, t) \mathrm{d} x=t
$$

In a transition region at the contact line near $x=1$, we write

$$
x=1+\psi c(t)+\varphi d(t) \eta, \quad h=\delta H,
$$

where we take $d=\dot{c}^{-1 / 3}$ for convenience in subsequent manipulations, and $\psi=\psi(\delta)$ and $\varphi=\varphi(\delta)$ are anticipated to be small parameters, since the contact line is expected to be close to $x=1$. Noting that the spatial and time derivatives transform to

$$
\frac{\partial}{\partial t} \rightarrow \frac{\partial}{\partial t}-\left(\frac{\psi \dot{c}}{\varphi d}+\frac{\eta \dot{d}}{d}\right) \frac{\partial}{\partial \eta}, \quad \frac{\partial}{\partial x} \rightarrow \frac{1}{\varphi d} \frac{\partial}{\partial \eta}
$$

after rearranging, equation (2.23) becomes

$$
\delta d^{4} \frac{\partial H}{\partial \bar{t}}-\left(\frac{\delta \psi}{\varphi}+\eta \delta \dot{d} d^{3}\right) \frac{\partial H}{\partial \eta}+\frac{\delta^{4}}{3 \varphi^{4}} \frac{\partial}{\partial \eta}\left(H^{3} \frac{\partial^{3} H}{\partial \eta^{3}}\right)=d^{4} \Theta(-\psi c-\varphi d \eta) .
$$

In order to obtain a non-trivial dominant balance in (3.20), we balance the second and fourth terms, which requires

$$
\psi=\frac{\delta^{3}}{\varphi^{3}}, \quad \varphi \ll \psi \quad \text { as } \quad \delta \rightarrow 0 .
$$

The second condition tells us that for $O(1)$ time, in the limit $\delta \rightarrow 0$, the apparent contact line moves by an amount that is much greater in extent than the width of the transition region. Thus, we may ignore injection in the transition region, and the corresponding 
equation for $H$ becomes

$$
-\frac{\partial H}{\partial \eta}+\frac{1}{3} \frac{\partial}{\partial \eta}\left(H^{3} \frac{\partial^{3} H}{\partial \eta^{3}}\right)=0 .
$$

Analysing the behaviour of (3.22) as $\eta \rightarrow-\infty$, matching it to the outer solution (3.12), as has been done in King \& Bowen (2001), and using (3.21), we obtain the necessary scalings for $\psi$ and $\varphi$, namely

$$
\psi=\frac{1}{\log (1 / \delta)}, \quad \varphi=\delta \log ^{1 / 3}(1 / \delta) .
$$

We immediately note that (3.21) hold true. These scalings will be further verified by the numerical solutions presented in $\S 4$. Thus, we conclude that for $O(1)$ time, the contact line is indeed stationary to leading order in $\delta$, and moves a distance $O(1 / \log (1 / \delta))$ at next order.

\subsection{Late-Time Behaviour}

To explore the late-time behaviour of the system, we will rescale both time and the film thickness appropriately to arrive at a limit in which the surface-tension term in $(2.23)$ is the only dominant term at leading order in the relevant asymptotic parameter (which will be determined as part of the analysis). This leading-order problem constitutes the solution in an outer region, the region to the left of the moving apparent contact line (see figure 3). At this stage, though, the position of the apparent contact line is an unknown function of time. We will solve for the film thickness in the transition region near the apparent contact line and match the solutions from the outer, transition and precursor-layer regions to obtain the full profile of the film thickness and the leadingorder solution for the location of the moving boundary $a(t)$. We will also see at the end of this section, and in $\S 5$, that the late-time behaviour of our model is a generalization of the classical droplet-spreading problem when we allow the volume of liquid to be time dependent. However, unlike the classical droplet-spreading problem, we will obtain unbounded growth of the film, which is entirely due to the finite-width injection region. In $\S 5$ we will consider some generalizations to our injection-spreading problem, and we will see that unbounded growth is not always guaranteed and depends on the functional form of the injection rate.

\subsubsection{The Outer Region}

Unlike the spreading-droplet scenario considered in King \& Bowen (2001), here we anticipate that the injection results in a film thickness that is much larger than $\delta$ for late time. Thus, we scale

$$
t=\frac{\bar{t}}{\lambda}, \quad h=\frac{\bar{h}}{\lambda},
$$

where $\lambda=\lambda(\delta) \ll 1$ is to be determined. We note that we do not scale $x$, because we anticipate that $a$ will move by an $O(1)$ amount on this timescale. After rearranging, (2.23) becomes

$$
\lambda^{4} \frac{\partial \bar{h}}{\partial \bar{t}}+\frac{1}{3} \frac{\partial}{\partial x}\left(\bar{h}^{3} \frac{\partial^{3} \bar{h}}{\partial x^{3}}\right)=\lambda^{4} \Theta(1-x) .
$$

The surface-tension term in (3.25) is dominant to leading order in $\lambda^{4}$. We assume $\delta \ll \lambda^{4} \ll 1$ as $\delta \rightarrow 0$, and will verify this assertion a posteriori. Expanding the dependent 


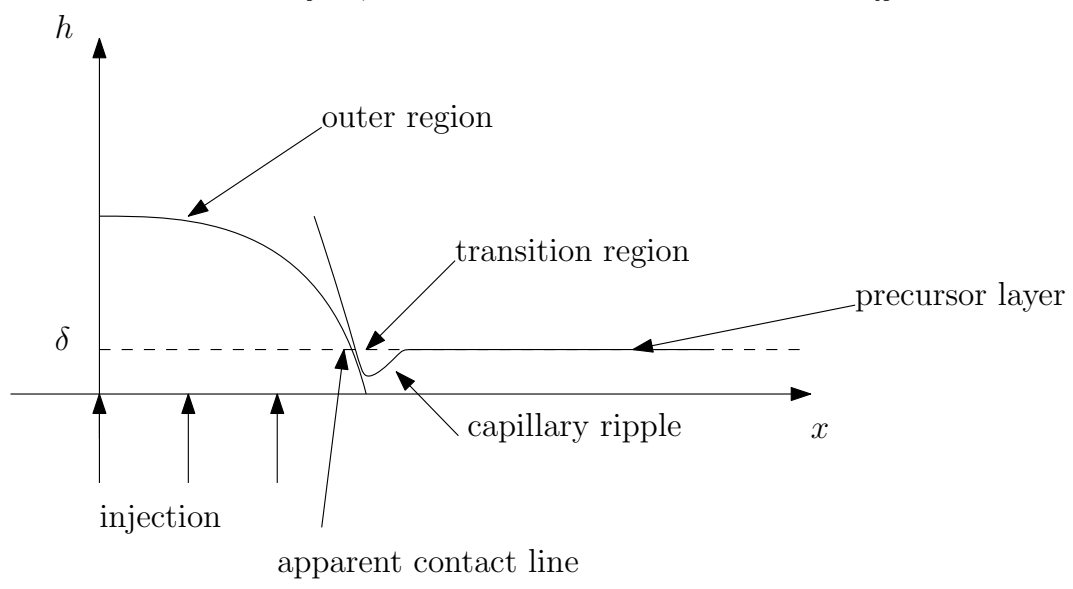

Figure 3: A schematic diagram of the outer, transition and precursor layer regions, and the position of the apparent contact line. The film thicknesses are not drawn to scale.

variables as

$$
\begin{array}{rlrl}
\bar{h}(x, \bar{t}) & \sim \bar{h}_{0}(x, \bar{t})+\lambda^{4} \bar{h}_{1}(x, \bar{t})+\cdots & \text { as } & \lambda \rightarrow 0, \\
a(\bar{t}) \sim a_{0}(\bar{t})+\lambda^{4} a_{1}(\bar{t})+\cdots & \text { as } & \lambda \rightarrow 0,
\end{array}
$$

the leading-order problem becomes

$$
\frac{\partial}{\partial x}\left(\bar{h}_{0}^{3} \frac{\partial^{3} \bar{h}_{0}}{\partial x^{3}}\right)=0,
$$

with

$$
\begin{aligned}
& \frac{\partial \bar{h}_{0}}{\partial x}=\frac{\partial^{3} \bar{h}_{0}}{\partial x^{3}}=0 \quad \text { at } \quad x=0, \\
& \bar{h}_{0}=0 \quad \text { at } \quad x=a_{0}(\bar{t}) .
\end{aligned}
$$

The leading-order version of the conservation-of-mass equation (2.27) becomes

$$
\bar{t}=\int_{0}^{a_{0}(\bar{t})} \bar{h}_{0}(x, \bar{t}) \mathrm{d} x .
$$

Equation (3.28) with conditions (3.29)-(3.31) has a parabolic solution, namely,

$$
\bar{h}_{0}(x, \bar{t})=\frac{3 \bar{t}\left(a_{0}^{2}-x^{2}\right)}{2 a_{0}^{3}},
$$

where $a_{0}(\bar{t})$ is unknown. In order to determine $a_{0}(\bar{t})$, we will explore the solution in the transition region near the moving boundary and then match to (3.32).

\subsubsection{The Transition Region}

In the transition region near the moving front, we write

$$
t=\frac{\bar{t}}{\lambda}, \quad x=a_{0}(\bar{t})+\nu s(\bar{t}) \xi, \quad h=\delta H
$$


where $\nu \ll 1$, and we set $s(\bar{t})=\dot{a}_{0}^{-1 / 3}$ for convenience in subsequent manipulations. With this change of variables, the spatial and time derivatives transform to

$$
\frac{\partial}{\partial \bar{t}} \rightarrow \frac{\partial}{\partial \bar{t}}-\left(\frac{\dot{a}_{0}}{\nu s}+\frac{\xi \dot{s}}{s}\right) \frac{\partial}{\partial \xi}, \quad \frac{\partial}{\partial x} \rightarrow \frac{1}{\nu s} \frac{\partial}{\partial \xi}
$$

Furthermore, from $\S 3.2$, we know that a moves $O(1 / \log (1 / \delta))$ away from the end of the injection region for $O(1)$ time. Therefore, assuming that $\nu \ll 1 / \log (1 / \delta)$ as $\delta \rightarrow 0$ (to be verified a posteriori), the transition region will lie outside the injection region for late time, and equation (2.23) becomes

$$
\lambda\left(s^{4} \frac{\partial H}{\partial \bar{t}}-\left(\frac{1}{\nu}+\xi \dot{s} s^{3}\right) \frac{\partial H}{\partial \xi}\right)+\frac{\delta^{3}}{3 \nu^{4}} \frac{\partial}{\partial \xi}\left(H^{3} \frac{\partial^{3} H}{\partial \xi^{3}}\right)=0 .
$$

In order to obtain a non-trivial dominant balance in (3.35), we balance the second and fourth terms, which requires that

$$
\nu=\frac{\delta}{\lambda^{1 / 3}} .
$$

After rearranging, (3.35) then becomes

$$
\nu\left(s^{4} \frac{\partial H}{\partial \bar{t}}-\xi \dot{s} s^{3} \frac{\partial H}{\partial \xi}\right)-\frac{\partial H}{\partial \xi}+\frac{1}{3} \frac{\partial}{\partial \xi}\left(H^{3} \frac{\partial^{3} H}{\partial \xi^{3}}\right)=0 .
$$

We expand the dependent variables in powers of $\nu=\nu(\delta)$, assuming $\delta \ll \nu \ll 1$ as $\delta \rightarrow 0$, which we will also check a posteriori, and, thus, we write

$$
H(\xi, \bar{t}) \sim H_{0}(\xi, \bar{t})+\nu H_{1}(\xi, \bar{t})+\cdots \quad \text { as } \quad \nu \rightarrow 0 .
$$

Substituting (3.38) into (3.37), retaining leading-order terms, integrating once, and applying the matching condition with the precursor layer

$$
H_{0} \rightarrow 1 \quad \text { as } \quad \xi \rightarrow \infty,
$$

we find that $H_{0}$ satisfies

$$
-H_{0}+\frac{1}{3} H_{0}^{3} \frac{\partial^{3} H_{0}}{\partial \xi^{3}}=-1 .
$$

To match with the outer solution, we need to understand the behaviour of the transition-region solution as $\xi \rightarrow-\infty$. We assume the solution is of the form $H_{0} \sim B(-\xi)^{m} \log ^{n}(-\xi)$, as $\xi \rightarrow-\infty$, for some values of $B, m$ and $n$. Choosing $m=1, n=1 / 3$, and $B=9^{1 / 3}$ gives the leading-order balance as $\xi \rightarrow-\infty$ between the two terms on the left-hand side of (3.40). Thus, our second matching condition is

$$
H_{0} \sim 9^{1 / 3}(-\xi) \log ^{1 / 3}(-\xi) \quad \text { as } \quad \xi \rightarrow-\infty .
$$

We note that (3.40) has been solved by Duffy \& Wilson (1997) for the case when the right-hand side is zero. Furthermore, they also obtain the asymptotic behaviour (3.41) (modulo the pre-factor), which confirms our result, since as $\xi \rightarrow-\infty, H_{0} \rightarrow \infty$, and the right-hand side of (3.40), which is constant, does not appear in the dominant balance. We solve for $H_{0}$ using a shooting method. Specifically, we expand $H_{0}$ using

$$
H_{0} \sim 1+\tilde{H}_{0} \quad \text { as } \quad \xi \rightarrow \infty,
$$

where $\tilde{H}_{0} \ll 1$. The equation for $\tilde{H}_{0}$ is

$$
\frac{1}{3} \frac{\partial^{3} \tilde{H}_{0}}{\partial \xi^{3}}-\tilde{H}_{0}=0
$$


which has a solution that is a combination of exponentials. Retaining only decaying solutions and appropriately choosing the origin, we may write the solution as

$$
H_{0} \sim 1+\tilde{A} \exp \left(-3^{1 / 3} \xi / 2\right) \cos \left(3^{5 / 6} \xi / 2\right) \quad \text { as } \quad \xi \rightarrow \infty .
$$

We then solve (3.40) by using $H_{0}, H_{0}^{\prime}, H_{0}^{\prime \prime}$ given by (3.44) evaluated at some large value of $\xi$ (we used $\xi=10$ ); $\tilde{A}$ is our shooting parameter. We note that we can restrict the interval for possible values for $\tilde{A}$ to, for example, $[1, \exp (2 \pi / \sqrt{3})]$ (McEwan \& Taylor 1966; Tuck \& Schwartz 1990). We vary $\tilde{A}$ in order to match the behaviour as $\xi \rightarrow-\infty$ specified by (3.41). As in Tuck \& Schwartz (1990), we use the fact that $H_{0} H_{0}^{\prime} H_{0}^{\prime \prime} \rightarrow-3$ as $\xi \rightarrow-\infty$ to provide us with an accurate indicator of how close we are to the required solution.

\subsubsection{Matching}

We first match the transition region with the outer region. We write (3.41) in outer variables as

$$
\begin{aligned}
\bar{h}_{0} & \sim \frac{9^{1 / 3} \lambda^{4 / 3}\left(a_{0}-x\right) \log ^{1 / 3}\left(\lambda^{1 / 3}\left(a_{0}-x\right) /(\delta s)\right)}{s} \\
& \sim \frac{9^{1 / 3} \lambda^{4 / 3}\left(a_{0}-x\right) \log ^{1 / 3}(1 / \delta)}{s} \quad \text { as } \quad \delta \rightarrow 0 .
\end{aligned}
$$

The leading-order term in the outer solution (3.32) is

$$
\bar{h}_{0} \sim \frac{3 \bar{t}\left(a_{0}-x\right)}{a_{0}^{2}} \quad \text { as } \quad x \rightarrow a_{0}^{-} .
$$

This suggests that, to match (3.45) and (3.46), the appropriate scaling for $\lambda$ should be

$$
\lambda=\frac{1}{\log ^{1 / 4}(1 / \delta)} .
$$

Thus, (3.36) gives

$$
\nu=\delta \log ^{1 / 12}(1 / \delta) .
$$

We note that this confirms the hypotheses that $\delta \ll \nu \ll \lambda^{4} \ll \lambda \ll 1$, as $\delta \rightarrow 0$. Logarithmic scales also appear in similar problems described in Lacey (1982), Hocking (1983), and King \& Bowen (2001), for example. However, for the classical problem of a spreading droplet, the relevant scale is $1 / \log (1 / \delta)$ (King \& Bowen 2001), which is asymptotically smaller (in the limit $\delta \rightarrow 0$ ) than our result (3.47) in the injection case. Matching (3.45) with (3.46), we obtain

$$
\dot{a}_{0}=\frac{3 \bar{t}^{3}}{a_{0}^{6}},
$$

which can be solved to determine $a_{0}(\bar{t})$. We also remark that (3.49) is a version of Tanner's law, since it follows that, at the contact line,

$$
\frac{\partial \bar{h}_{0}}{\partial x}=-\left(9 \dot{a}_{0}\right)^{\frac{1}{3}}
$$

We match the late-time behaviour for the contact line with the behaviour for $O(1)$ time, which results in imposing the initial condition $a_{0} \rightarrow 1$ as $t \rightarrow 0$. Thus, (3.49) can be solved to yield

$$
a_{0}(\bar{t})=\left(1+\frac{21 \bar{t}^{4}}{4}\right)^{1 / 7}
$$


and so,

$$
\bar{h}_{0}(0, \bar{t})=\frac{3 \bar{t}}{2 a_{0}}=\frac{3 \bar{t}}{2}\left(1+\frac{21 \bar{t}^{4}}{4}\right)^{-1 / 7} .
$$

In terms of the original variables, $h_{0}=\bar{h}_{0} / \lambda$ and $t=\bar{t} / \lambda$,

$$
a_{0}(t)=\left(1+\frac{21 \lambda^{4} t^{4}}{4}\right)^{1 / 7}, \quad h_{0}(0, t)=\frac{3 t}{2}\left(1+\frac{21 \lambda^{4} t^{4}}{4}\right)^{-1 / 7} \text { for } \quad t=O(1 / \lambda) .
$$

We observe that, for late time, the apparent contact line moves much faster $\left(\sim t^{4 / 7}\right)$ than the classical non-injection case $\left(\sim t^{1 / 7}\right)$ considered in King \& Bowen (2001). We also note that, through $\lambda$, the leading-order results (3.53) have a dependence on the precursor thickness, $\delta$. In physical systems where precursor layers are not observed, $\delta$ can be treated as an adjustable parameter to be determined from experimental data.

As mentioned at the beginning of $\S 3$, we can generalize the results from the classical droplet-spreading problem (see, for example, Voinov 1976; Cox 1986; Bonn et al. 2009) to the case of a time-dependent injection rate using the Cox-Voinov law (Bonn et al. 2009) to obtain the full late-time $(t \gg 1 / \lambda)$ behaviour of the film spreading (see Appendix $\mathrm{C}$ for details).

We further note that we can also obtain the power-law dependence on time in (3.53) using scaling arguments. The matching condition (3.49) can also simply be deduced from a comparison of the slopes of $h$ in the inner and outer regions obtained from (3.45) and (3.46), respectively. Scaling $x \sim a$, the slope of the outer solution $\sim h / a \sim t / a^{2}$ from (3.32), while the inner region has height $\sim \delta$ and length $\sim \delta \dot{a}^{-1 / 3}$ from (3.33) (with $\lambda=1$ ) and, thus, has slope $t / a^{2} \sim \dot{a}^{1 / 3}$ confirming the scalings $a \sim t^{4 / 7}$ and $h \sim t^{3 / 7}$. Furthermore, if we look for a similarity solution to the problem for late time (essentially, away from the injection region) of the form $t^{\alpha} f\left(x / t^{\beta}\right)$, for some exponents $\alpha$ and $\beta$, we can recover $\alpha=3 / 7$ and $\beta=4 / 7$ and the parabolic profile for the outer solution. This, as we saw, is confirmed by the late-time asymptotic analysis. Thus, for late time the aspect ratio given by $h_{0}(0, t) / a_{0}(t) \sim t^{-1 / 7}$, so remains small for all time.

\section{Numerical Results and Discussion}

We now present numerical solutions to the system (2.23)-(2.26). We approximate the boundary condition (2.25) by imposing

$$
\begin{aligned}
& h=\delta \quad \text { on } \quad x=l, \\
& \frac{\partial h}{\partial x}=0 \quad \text { on } \quad x=l,
\end{aligned}
$$

where $l$ is some large number. We used $l=60$.

We employ a transformation of the coordinates that yields an adaptive mesh, which allows us to solve the problem for large time for a moderate computational cost (see Appendix A for details). We solve the problem using MATLAB with a linear implicit Euler scheme. In figure 4(a), we show the numerical solutions for the spatial profile of the thickness $h(x, t)$ for various times where we have imposed a dimensionless precursor-layer thickness of $\delta=10^{-3}$. As anticipated, the film thickness increases with time, and the front advances. We observe that the solutions appear self-similar, which indeed is the case for late time as found at the end of §3.3.3. In figure 4(b), we show a zoom-in of the height profile near the apparent contact line, where we see characteristic capillary ripples. In figure $4(c)$, we show the numerical solutions for the spatial profile of the thickness $h(x, t)$ 
for various early times with a zoom-in of the apparent contact line near $x=1$. We see that capillary ripples form on either side of $x=1$ and subsequently travel outwards in both directions. In $\S 3.1$, we formulated the boundary-layer problem (3.8)-(3.11) for the transition between the spatially uniform solutions for early time given in (3.6). In figure $4(d)$, we show a plot of the film height near $x=1$, found by solving (3.8)-(3.11) numerically, compared with the full numerical solution. We see good agreement between the two solutions.

In figure 5, we compare the thickness profile of the film at time $t=10$ with the asymptotic predictions for late time for the outer- and transition-region profiles, given by (3.32) and (3.40) respectively, for two different precursor-layer thicknesses. We see excellent agreement between the numerical solution and the asymptotic solution as $\delta$ decreases. We have shifted the asymptotic solution for the inner region to allow for a detailed comparison with the numerical solution near the capillary ripple. This is shown in the small panels in the figures. In general, the positions of $x=a$ for the outer and inner regions are expected to differ, because the corrections to the leading-order outer solution for $a$ are large compared to the length scale of the inner region. We note that the predicted convergence is quite slow since our asymptotic sequence for $h(x, t)$ is logarithmic in nature. This slow logarithmic convergence is also present when slip regularization is used (see, for example, Lacey 1982; Hocking 1983).

In figure 6 , we show $\log -\log$ plots of $h(0, t)$ and the position of the apparent contact line, $a(t)$, given by the numerical solution to $(2.23)-(2.26)$, together with our asymptotic predictions for early time, (3.6), and late time, (3.51)-(3.52). The asymptotic solutions match well for the early and late time behaviours, and, in fact, are valid for a much wider range of times than expected. We also note that the contact line does not move to leading order in $\delta$ for early time, as shown in $\S 3.1$, and moves $O(1 / \log (1 / \delta))$ for $O(1)$ time, as shown in $\S 3.2$.

\section{General Injection Rates}

In this section, we extend the model to the case of non-constant injection by replacing (2.4) with

$$
\hat{w}=\hat{f}(\hat{x}, \hat{t}) \Theta(L-\hat{x}) \quad \text { on } \quad \hat{z}=0,
$$

for some dimensional supply function $\hat{f}(\hat{x}, \hat{t})$.

\subsection{Power-Law Injection}

We first consider the case in which $\hat{f}$ has a power-law dependence in time, i.e., $\hat{f}=$ $\hat{t}^{k} \hat{g}(\hat{x})$, where $\hat{g}$ is some integrable function on $[0,1]$, and $k$ is some real exponent whose range we will specify later on. We expect that $k<0$ for physically sensible models, such as a chemical reaction whose rate decreases with time. However, we will also study the case $k>0$. We note that, for $-1<k<0$, the injection rate is singular at $t=0$, but the volume of liquid (which is the time integral of the flux) is still finite. The case $k \leqslant-1$ corresponds to an infinite volume of liquid injected at $t=0$, which we exclude on physical grounds. For simplicity, in the following analysis we assume that $\hat{g}=W$ is constant, even though more general spatial variance could be incorporated using the same approach. Thus, the boundary condition on the surface $\hat{z}=0$ becomes

$$
\hat{w}=W \hat{t}^{k} \Theta(L-\hat{x}) \quad \text { on } \quad \hat{z}=0 .
$$



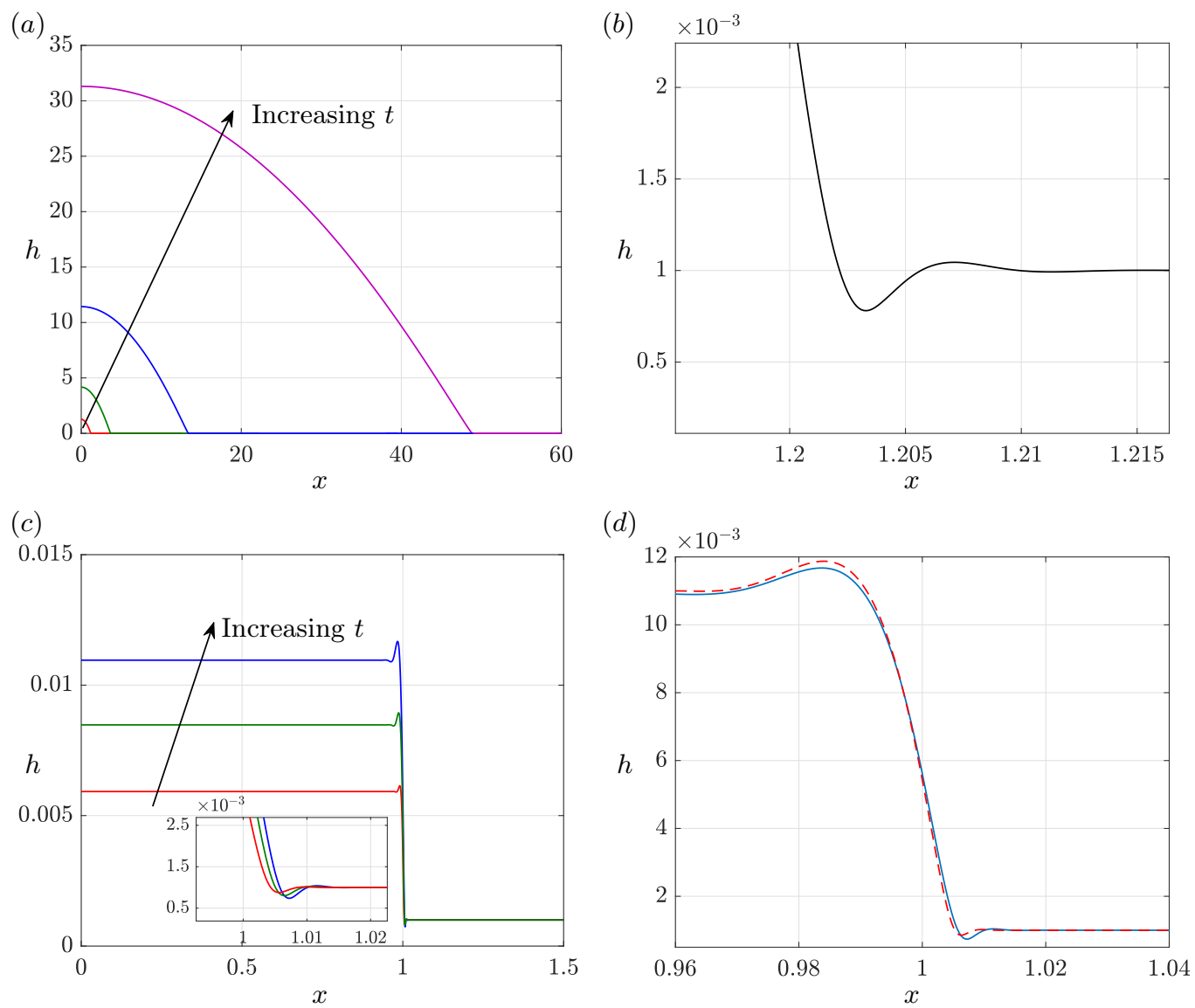

Figure 4: Plots of the film thickness, $h(x, t)$, satisfying (2.23)-(2.26) (a) at $t=1$ (red), $t=10$ (green), $t=100$ (blue), $t=1000$ (purple), (b) near $h=\delta$ and $x=1.2$ for $t=1$, and $(c)$ at $t=0.005$ (red), $t=0.0075$ (green), $t=0.01$ (blue). In $(d)$, we show the film thickness (solid blue) along with the solution to (3.8)-(3.11) (dashed red) near $x=1$ for $t=0.01$. In all figures, $\delta=10^{-3}$.

In this case, we require a different non-dimensionalization and we use

$$
\begin{gathered}
(\hat{x}, \hat{a})=L(x, a), \quad(\hat{z}, \hat{h})=\left(\mu W^{1 /(k+1)} L^{4} / \gamma\right)^{(k+1) /(3 k+4)}(z, h), \\
\hat{u}=\left(\gamma W^{3} L^{3 k} / \mu\right)^{1 /(3 k+4)} u, \quad \hat{w}=\left(\mu W^{4 / k} L^{4} / \gamma\right)^{k /(3 k+4)} w \\
\hat{t}=\left(\mu L^{4} / \gamma W^{3}\right)^{1 /(3 k+4)} t, \quad \hat{p}=\left(\mu^{k+1} \gamma^{2 k+3} W / L^{2 k+4}\right)^{1 /(3 k+4)} p .
\end{gathered}
$$

We assume a long and thin geometry, i.e., that the aspect ratio

$$
\epsilon=\left(\mu W^{1 /(k+1)} L^{k / k+1} / \gamma\right)^{(k+1) /(3 k+4)} \ll 1,
$$

and that we have a thin precursor film, i.e., $\delta=h_{\infty} /(\epsilon L) \ll 1$. Following the same steps as in $\S 2.2$, we manipulate the dimensionless versions of $(2.1)-(2.3),(2.5)-(2.7)$ and $(5.2)$ 
16

(a)

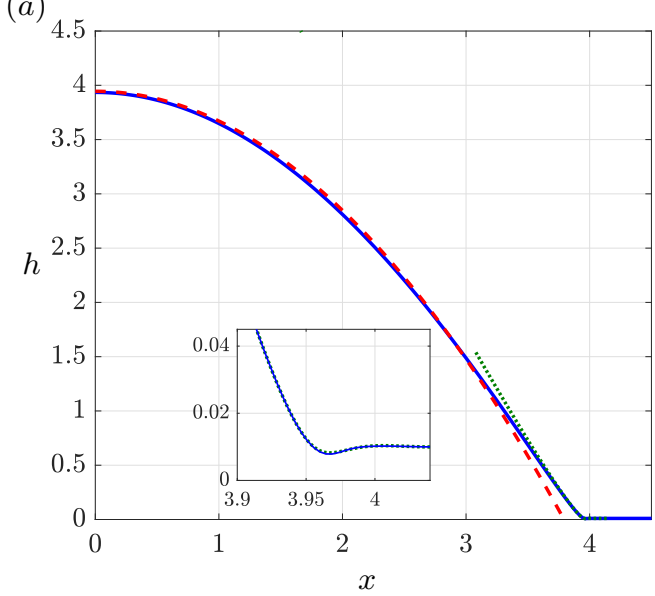

(b)

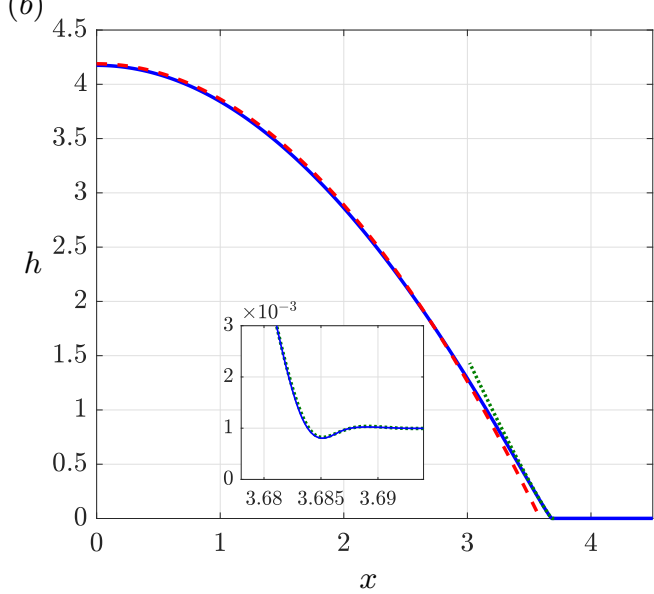

Figure 5: Plots of the film thickness, $h$, satisfying (2.23)-(2.26), at $t=10$ : numerical solution (solid blue), asymptotic solution in the outer region (3.32) (dashed red), asymptotic solution in the transition region (3.40) (dotted green) for $(a) \delta=10^{-2}$ and (b) $\delta=10^{-3}$.

(a)

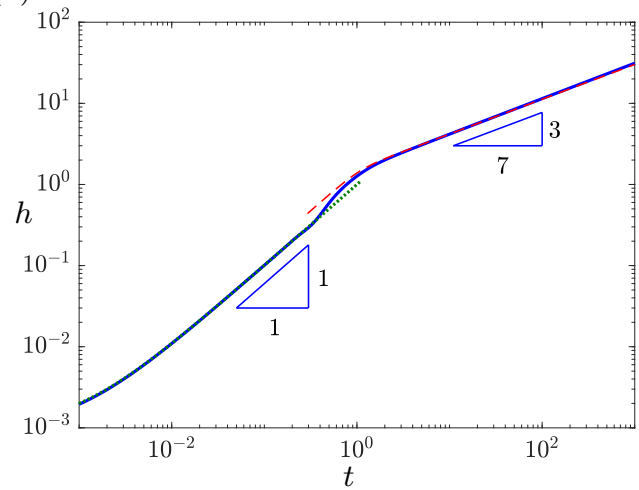

(b)

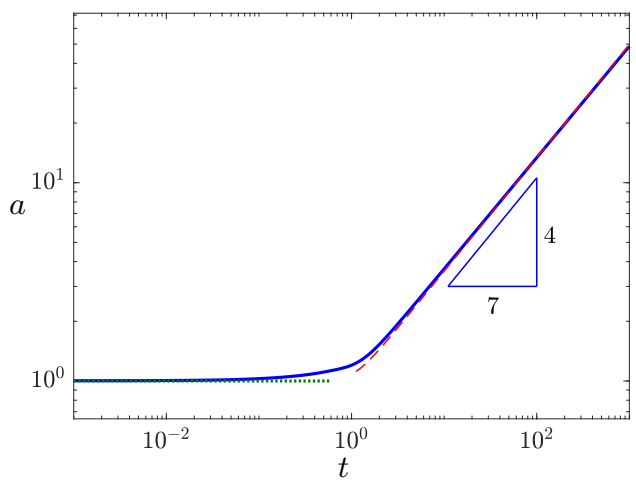

Figure 6: Log-log plots of the numerical solution to (2.23)-(2.26) (solid blue) for $(a)$ $h(0, t)$ and $(b) a(t)$. The asymptotic predictions for early time, (3.6) (dotted green), and late time, (3.51)-(3.52) (dashed red) are also shown. Here, $\delta=10^{-3}$.

and we arrive at the evolution equation for $h$, namely

$$
\frac{\partial h}{\partial t}+\frac{1}{3} \frac{\partial}{\partial x}\left(h^{3} \frac{\partial^{3} h}{\partial x^{3}}\right)=t^{k} \Theta(1-x)
$$

together with the boundary conditions given by (2.24)-(2.26).

For early time, we use a scaling similar to (3.1), but take account of the exponent $k$ in the injection function by setting

$$
h=\delta \tilde{h}, \quad t=\delta^{1 /(k+1)} \tilde{t},
$$

where, here, we restrict ourselves to considering $k>-1$ so that the scalings are valid for early time. After asymptotically expanding $h$ and $a$ in powers of $\delta$ as before, we arrive at 
a dominant balance between the first and third term in (5.5). Using the same notation as in $\S 3$, we obtain

$$
\tilde{h}_{0}(x, \tilde{t})=1+\frac{\tilde{t}^{k+1}}{k+1} \Theta(1-x) .
$$

As in $\S 3.1$, it is possible to obtain a problem for the boundary layer near $x=1$, analogous to (3.8)-(3.11), which smoothly joins the two solutions given in (5.7), but we do not pursue that here. Instead, we point out that we can consider two distinct temporal regimes in which more analytical progress can be made (see Appendix B for the details of this analysis).

For late time, we scale

$$
h=\frac{\bar{h}}{\lambda_{k}}, \quad t=\frac{\bar{t}}{\lambda_{k}^{1 /(k+1)}},
$$

where we assume $\lambda_{k} \ll 1$. Repeating the analysis in $\S 3.3$, we find the appropriate choice of $\lambda_{k}$ is $(c f .(3.47))$

$$
\lambda_{k}=\frac{1}{\log ^{(k+1) /(3 k+4)}(1 / \delta)},
$$

which is indeed small for $k>-1$. Therefore, we obtain the following leading-order results for the position of the apparent contact line and the maximum film thickness

$$
\begin{aligned}
a_{0}(\bar{t}) & =\left(1+\frac{21 \bar{t}^{3 k+4}}{(k+1)^{3}(3 k+4)}\right)^{1 / 7}, \\
\bar{h}_{0}(0, \bar{t}) & =\frac{3 \bar{t}^{k+1}}{2(k+1) a_{0}}=\frac{3 \bar{t}^{k+1}}{2(k+1)}\left(1+\frac{21 \bar{t}^{3 k+4}}{(k+1)^{3}(3 k+4)}\right)^{-1 / 7} .
\end{aligned}
$$

In an analogous way to $\S 3$, we can use the Cox-Voinov law to obtain the full late-time behaviour of the film spreading when $t \gg 1 / \lambda_{k}^{1 /(k+1)}$.

We note that, in the case when $k=0$, we recover the results (3.51) and (3.52). Furthermore, when $k=-3 / 4, \bar{h}_{0}$ tends to a constant, i.e., injection is just enough to balance the surface-tension flow. Consequently, for $-1<k<-3 / 4$, injection can no longer support a growing or constant film thickness, and, thus, the film thickness decreases at large time. For very large time, we expect capillarity to force the film to approach the thickness of the precursor layer again, and, thus, using the scaling from (5.11), $h \sim t^{(4 k+3) / 7}$ gives $t=\delta^{7 /(4 k+3)} t^{\star}$ as the required scaling for $t$. We note that the same scaling can be derived from the balance $h-\delta \sim t^{k+1} / x, x \sim\left(\delta^{3} t\right)^{1 / 4}$ between the growth and the surface-tension term in (5.5). We do not pursue this situation further.

In figure 7 , we show log-log plots of the numerical solution and our asymptotic predictions for the maximum film thickness at the origin and the position of the apparent contact line as functions of time for different values of $k$. We see a good agreement between the numerical solution and the asymptotic results for early and late time.

Additionally, we observe that the aspect ratio, given by $h_{0}(0, t) / a_{0}(t) \sim t^{(k-1) / 7}$, grows in time if $k>1$. In this case, for late time, the thin-film hypothesis will eventually break down.

Finally, we note that the results (5.10)-(5.11) can be used in a simple inverse problem, in which we can determine the exponent of the injection rate, assuming it has a power-law dependence, by observing the spreading of the film front or the evolution of its maximum thickness at the origin for late time. Thus, if the film front is observed to spread like $t^{\sigma}$ for some exponent $\sigma$, then the exponent of the injection rate is given by $k=(7 \sigma-4) / 3$. 
(a)

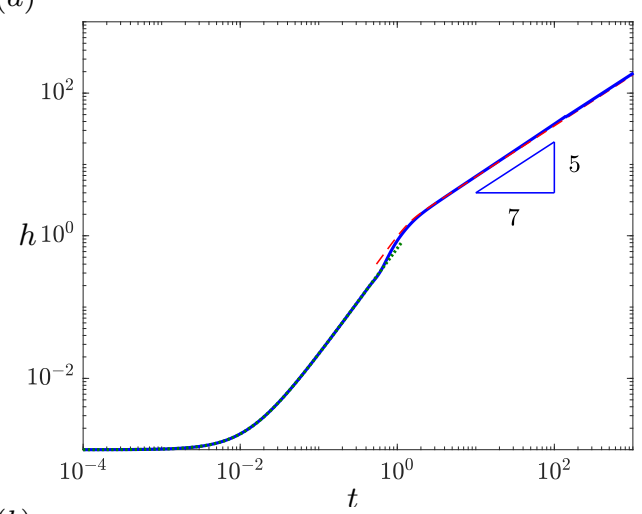

(b)

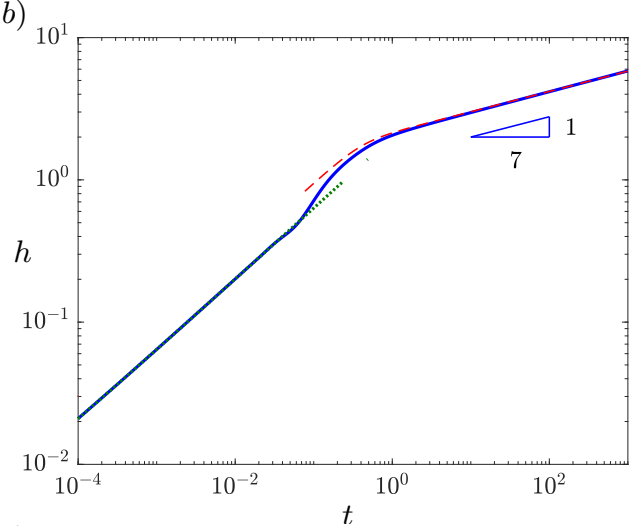

(c)

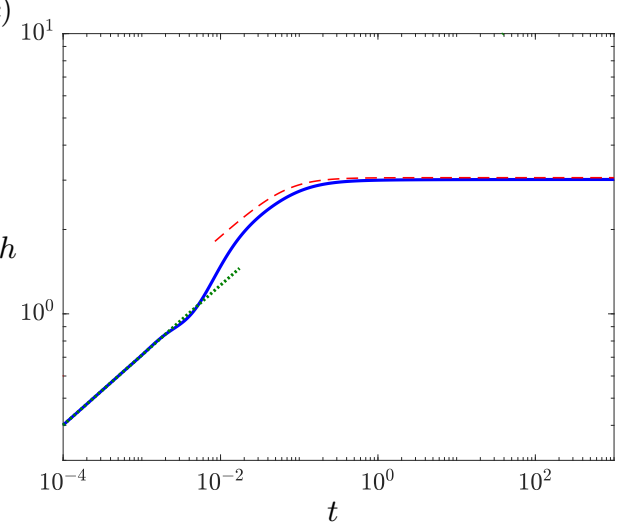

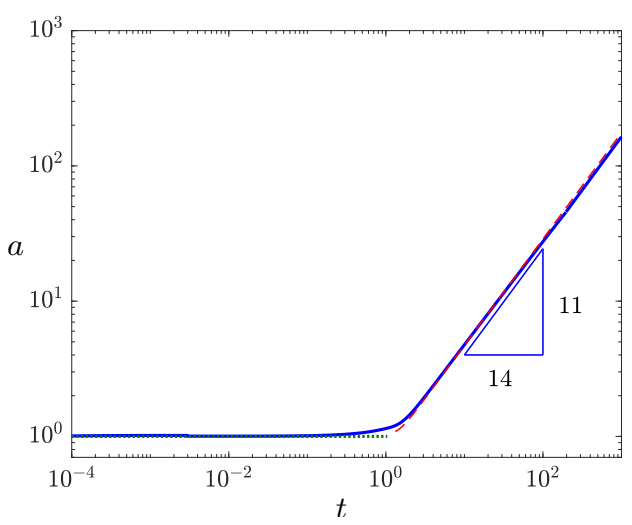
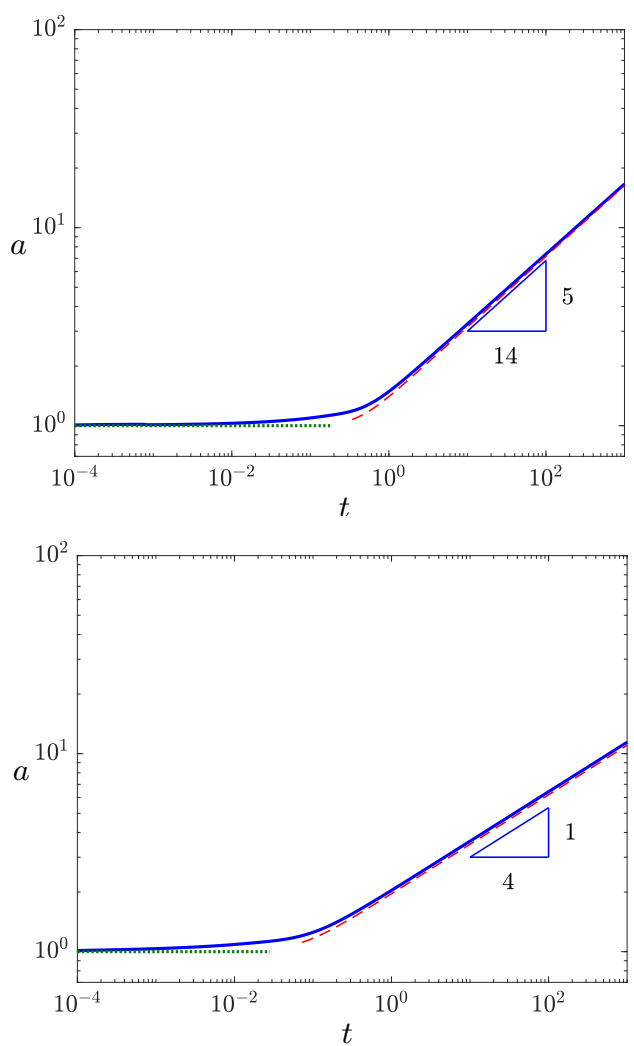

Figure 7: $\log -\log$ plots of the numerical solution to (5.5) with (2.24)-(2.26) (solid blue) for $h(0, t)$ (left column) and $a(t)$ (right column) for $(a) k=1 / 2,(b) k=-1 / 2,(c)$ $k=-3 / 4$. The asymptotic predictions for early time, (5.7) (dotted green), and late time, (5.10)-(5.11) (dashed red) are also shown. In all figures, $\delta=10^{-3}$.

For example, if experimental data reveals a linear spread of the film front, then the liquid is also injected linearly in time.

\subsection{Point-Source Injection}

We now consider the case when liquid is injected at the origin, i.e., a point-source injection with $\hat{f}=Q \delta(\hat{x})$ in (5.1), where $Q$ is a given liquid flux. We note that, due 
to the definition of the delta function, $Q=2 W L$ in terms of the notation in $\S 3$. The boundary condition on $\hat{z}=0$ then becomes

$$
\hat{w}=Q \delta(\hat{x}) \quad \text { on } \quad \hat{z}=0 .
$$

We non-dimensionalize using

$$
\begin{gathered}
(\hat{x}, \hat{a})=\left(\gamma Q^{3} \tau^{4} / \mu\right)^{1 / 7}(x, a), \quad(\hat{z}, \hat{h})=\left(\mu Q^{4} \tau^{3} / \gamma\right)^{1 / 7}(z, h), \\
\hat{u}=\left(\gamma Q^{3} / \mu \tau^{3}\right)^{1 / 7} u, \quad \hat{w}=\left(\mu Q^{4} / \gamma \tau^{4}\right)^{1 / 7} w, \quad \hat{t}=\tau t, \quad \hat{p}=\left(\mu^{3} \gamma^{4} / Q^{2} \tau^{5}\right)^{1 / 7} p,
\end{gathered}
$$

where we can choose the timescale $\tau=\left(h_{\infty}^{7} \gamma / \mu Q^{4}\right)^{1 / 3}$ so that the dimensionless precursor-layer thickness $\delta=h_{\infty}\left(\mu Q^{4} \tau^{3} / \gamma\right)^{-1 / 7}=1$ and we remove all dimensionless parameters from the model. However, we are interested in the situation where the film thickness is much larger than the precursor thickness and a long timescale over which the aspect ratio $\epsilon=\left(\mu^{2} Q / \gamma^{2} \tau\right)^{1 / 7} \ll 1$ so that the thin-film hypothesis is valid. Thus, we rescale our problem using

$$
t \rightarrow T t, \quad(x, a) \rightarrow T^{4 / 7}(x, a), \quad(z, h) \rightarrow T^{3 / 7}(z, h)
$$

for some $T \gg 1$. The evolution equation for $h$ becomes

$$
\frac{\partial h}{\partial t}+\frac{1}{3} \frac{\partial}{\partial x}\left(h^{3} \frac{\partial^{3} h}{\partial x^{3}}\right)=\delta(x),
$$

together with (2.24)-(2.26), in which the dimensionless precursor thickness now appears as $\delta=1 / T^{3 / 7} \ll 1$, and where we have used the fact that $\delta(\kappa x)=\delta(x) / \kappa$ for any $\kappa>0$. We note that, the thin-film hypothesis will hold as long as the new aspect ratio $\epsilon=\left(\mu^{2} Q / \gamma^{2} \tau T\right)^{1 / 7} \ll 1$, which is true for sufficiently large $T$. The appropriate limit of the late-time analysis presented in $\S 3.3$ holds in this case too, since the width of the slot is asymptotically small for late time, and so, can be described by a delta function. We, thus, recover the power-law dependencies

$$
\begin{aligned}
a_{0}(\bar{t}) & =\left(\frac{21}{32}\right)^{1 / 7} \bar{t}^{4 / 7}, \\
\bar{h}_{0}(0, \bar{t}) & =\frac{3}{4}\left(\frac{32}{21}\right)^{1 / 7} \bar{t}^{3 / 7},
\end{aligned}
$$

where, again, we can use the Cox-Voinov law, as mentioned in $\S 3$, to obtain the full late-time behaviour. However, we note that the numerical pre-factors are not the same as those in (3.51) and (3.52), since the strength of the source in this case is half that considered in $\S 3.3$.

In figure 8, we show log-log plots of the maximum film thickness and the position of the apparent contact line as functions of time for the case of point-source injection. To simulate the behaviour of the delta function, we have used two approaches. First, we approximate a point-source injection using the original numerical solution with a finite injection slot of small width $\hat{L}_{s}=\left(\gamma Q^{3} \tau^{4} / \mu\right)^{1 / 7} L_{s}$, keeping the total flux of liquid constant by increasing the injection rate, i.e., replacing (5.15) with

$$
\frac{\partial h}{\partial t}+\frac{1}{3} \frac{\partial}{\partial x}\left(h^{3} \frac{\partial^{3} h}{\partial x^{3}}\right)=\frac{\Theta\left(L_{s}-x\right)}{2 L_{s}},
$$

where $L_{s} \ll 1$. Second, we prescribe the flux at the origin by solving the problem 
(a)

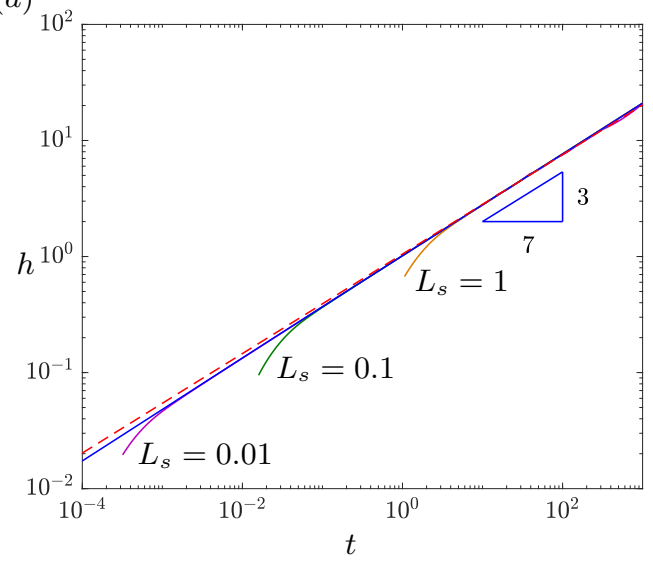

(b)

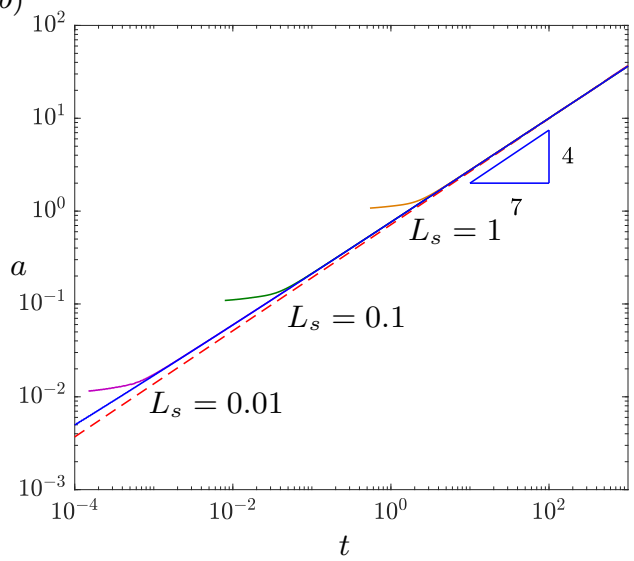

Figure 8: Log- $\log$ plots of the numerical solution to (5.19) with (5.20) and (2.25)-(2.26) (solid blue), the numerical solutions to (5.18) with (2.24)-(2.26) with $L_{s}=1$ (orange), $L_{s}=0.1$ (green), and $L_{s}=0.01$ (purple) for $(a) h(0, t)$ and $(b) a(t)$, compared with the asymptotic predictions for late time (3.53) (dashed red). Here, $\delta=10^{-3}$.

$$
\frac{\partial h}{\partial t}+\frac{1}{3} \frac{\partial}{\partial x}\left(h^{3} \frac{\partial^{3} h}{\partial x^{3}}\right)=0,
$$

subject to $(2.25),(2.26)$, and

$$
\frac{\partial h}{\partial x}=0, \quad \frac{1}{3} h^{3} \frac{\partial^{3} h}{\partial x^{3}}=\frac{1}{2} \quad \text { at } \quad x=0 .
$$

We also plot the asymptotic results (5.16) and (5.17) across the entire $t$ range (despite them only being formally valid for $t \gg 1$ ). We see a very good agreement between the numerical results obtained using the second approach and the asymptotic results for a wider range of time than anticipated. The asymptotic results provide a good estimate at early time, because, in this case, the slot has been shrunk to a point. For early time, we observe a slight discrepancy between the numerical and the asymptotic results, which can be explained by the finite precursor film, which is present everywhere on the substrate at $t=0$ and is not reflected by our "late-time" results. We also see that, as time increases, the numerical solution from the first approach converges to the numerical solution from the second approach (as anticipated) and to the asymptotic predictions (5.16) and (5.17), and the time to convergence decreases as $L_{s}$ decreases. To illustrate this, we consider the early-time behaviour. As before, we determine the linear growth for early time by balancing the first and third terms in (5.18), which gives

$$
h=\delta+\frac{t}{L_{s}} \Theta\left(L_{s}-x\right) .
$$

We obtain the range of validity of the early-time solution (5.21) by requiring that the "discontinuous" step that is initially at $x=L_{s}$ has not spread to the origin. By considering (5.21), we see that, if $t \ll \delta L_{s}$, then $h \sim \delta$, and the validity condition, found using a scaling argument on (5.19), reads

$$
\left(\delta^{3} t\right)^{1 / 4} \ll L_{s} .
$$


If $\delta L_{s} \ll t \ll 1$, then $h \sim t / L_{s}$ from (5.21), and the validity condition is instead

$$
\left(h^{3} t\right)^{1 / 4} \ll L_{s} .
$$

The two conditions (5.22) and (5.23) can be combined into a single range of validity, $t \ll \min \left\{L_{s}^{4} / \delta^{3}, L_{s}^{7 / 4}\right\}$. We note that when $L_{s}=1$, the validity condition becomes $t \ll 1$, as seen in $\S 3.1$.

\section{Thickness-Inhibited Injection}

A final regime of interest is one in which the rate of injection decreases as the liquid thickness grows. Such a scenario might arise when liquid forms through a chemical reaction on the surface, which requires the chemical to diffuse through the liquid film to reach the surface. This may be captured by considering a dimensional supply flux $W / \hat{h}^{q}$, where $q>0$ is a constant. We note that the case $q=1$ corresponds to standard diffusion with flux inversely proportional to the thickness of the film. The boundary condition on $\hat{z}=0$ then becomes

$$
\hat{w}=\frac{W}{\hat{h}^{q}} \Theta(L-\hat{x}) \quad \text { on } \quad \hat{z}=0 .
$$

This time, we non-dimensionalize (2.1)-(2.3), (2.5)-(2.7) and (6.1) using

$$
\begin{gathered}
(\hat{x}, \hat{a})=L(x, a), \quad(\hat{z}, \hat{h})=\left(\mu W L^{4} / \gamma\right)^{1 /(q+4)}(z, h), \\
\hat{u}=\left(\gamma^{q+1} W^{3} / \mu^{q+1} L^{3 q}\right)^{1 /(q+4)} u, \quad \hat{w}=\left(\gamma W^{4 / q} / \mu L^{4}\right)^{q /(q+4)} w, \\
\hat{t}=\left(\mu L^{4} / \gamma W^{3 /(q+1)}\right)^{(q+1) /(q+4)} t, \quad \hat{p}=\left(\mu \gamma^{q+3} W / L^{2 q+4}\right)^{1 /(q+4)} p .
\end{gathered}
$$

We continue assuming a long and thin geometry, i.e., that the aspect ratio

$$
\epsilon=\left(\mu W / \gamma L^{q}\right)^{1 /(q+4)} \ll 1
$$

and that the precursor layer is thin, i.e., $\delta=h_{\infty} /(\epsilon L) \ll 1$. The evolution equation for $h$ is then

$$
\frac{\partial h}{\partial t}+\frac{1}{3} \frac{\partial}{\partial x}\left(h^{3} \frac{\partial^{3} h}{\partial x^{3}}\right)=\frac{1}{h^{q}} \Theta(1-x),
$$

together with $(2.24)-(2.26)$.

For early time, we rescale the film thickness and time according to

$$
h=\delta \tilde{h}, \quad t=\delta^{q+1} \tilde{t} .
$$

The leading-order (in $\delta$ ) solution satisfies

$$
\frac{\partial \tilde{h}_{0}}{\partial \tilde{t}}=\frac{1}{\tilde{h}_{0}^{q}} \Theta(1-x)
$$

with an initial condition given by

$$
\tilde{h}_{0}=1 \quad \text { at } \quad \tilde{t}=0 .
$$

The solution to (6.6) with (6.7) has a power-law behaviour in $0 \leqslant x \leqslant 1$ and is constant in $x>1$, with

$$
\tilde{h}_{0}(x, \tilde{t})=(1+(q+1) \tilde{t} \Theta(1-x))^{1 /(q+1)} .
$$

A boundary-layer analysis near $x=1$ analogous to the one in $\S 3.1$ provides a continuous 
solution smoothly joining the film thicknesses on either side of $x=1$, but we do not examine it in this paper.

For late time, we scale

$$
h=\frac{\bar{h}}{\lambda_{q}}, \quad t=\frac{\bar{t}}{\lambda_{q}^{q+1}},
$$

where we assume $\lambda_{q} \ll 1$. Repeating the analysis in $\S 3.3$, we find the appropriate choice of $\lambda_{q}$ is $(c f .(3.47))$

$$
\lambda_{q}=\frac{1}{\log ^{1 /(q+4)}(1 / \delta)},
$$

which is indeed small for $q>0$. We note that the analogue of (3.31) is

$$
V(\bar{t})=\int_{0}^{\bar{t}} \int_{0}^{1} \frac{1}{\bar{h}_{0}^{q}(x, s)} \mathrm{d} x \mathrm{~d} s=\int_{0}^{a_{0}(\bar{t})} \bar{h}_{0}(x, \bar{t}) \mathrm{d} x,
$$

where $V$ is the leading-order volume of the liquid.

Repeating the analysis in $\S 3.3$, we obtain the outer solution for the leading-order film thickness, $\bar{h}_{0}$

$$
\bar{h}_{0}(x, \bar{t})=A(\bar{t})\left(a_{0}^{2}-x^{2}\right)
$$

where $A(\bar{t})$ satisfies

$$
A(\bar{t})=\frac{3 V(\bar{t})}{2 a_{0}(\bar{t})^{3}}=\frac{3}{2 a_{0}(\bar{t})^{3}} \int_{0}^{\bar{t}} \int_{0}^{1} \frac{1}{A(s)^{q}\left(a_{0}^{2}(s)-x^{2}\right)^{q}} \mathrm{~d} x \mathrm{~d} s .
$$

In this case, we end up with the following integro-differential equation for $a_{0}(\bar{t})$

$$
\dot{a}_{0}=\frac{8}{9} a_{0}^{3} A^{3}=\frac{3 V^{3}}{a_{0}^{6}}=\frac{3}{a_{0}^{6}}\left(\int_{0}^{\bar{t}} \int_{0}^{1} \frac{1}{A(s)^{q}\left(a_{0}^{2}(s)-x^{2}\right)^{q}} \mathrm{~d} x \mathrm{~d} s\right)^{3} .
$$

We note that setting $q=0$ recovers (3.49).

In order to make further analytical progress, we first transform (6.14) into an ordinary differential equation by differentiating both sides with respect to time and using (6.13). We then obtain

$$
\ddot{a}_{0}=-6 \frac{\dot{a}_{0}^{2}}{a_{0}}+4 \chi(\bar{t})\left(\frac{9 \dot{a}_{0}}{8 a_{0}^{3}}\right)^{(2-q) / 3},
$$

where

$$
\chi(\bar{t})=\int_{0}^{1} \frac{1}{\left(a_{0}(\bar{t})^{2}-x^{2}\right)^{q}} \mathrm{~d} x .
$$

Anticipating that $a_{0} \gg 1$ for large $\bar{t}$, we can approximate $(6.15)$ by

$$
\ddot{a}_{0}=-6 \frac{\dot{a}_{0}^{2}}{a_{0}}+4\left(\frac{9 \dot{a}_{0}}{8 a_{0}^{3}}\right)^{(2-q) / 3} \frac{1}{a_{0}^{2 q}} .
$$

If we now try an ansatz of the form $a_{0}=C \bar{t}^{D}$, for some constants $C, D$, we find

$$
D=\frac{q+4}{4 q+7}, \quad C=\left(\frac{2^{3 q} 3^{1-2 q}(4 q+7)^{q+4}}{(q+4)^{q+1}(q+7)^{3}}\right)^{1 /(4 q+7)} .
$$

We, thus, obtain the power-law behaviour of the maximum film thickness and the position 
(a)

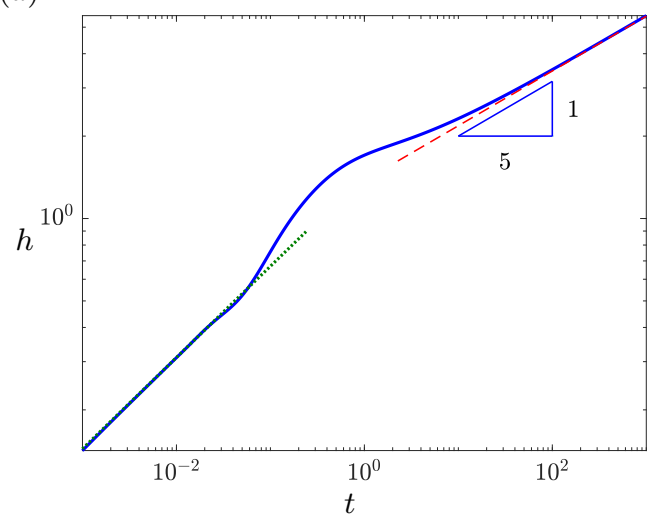

(b)

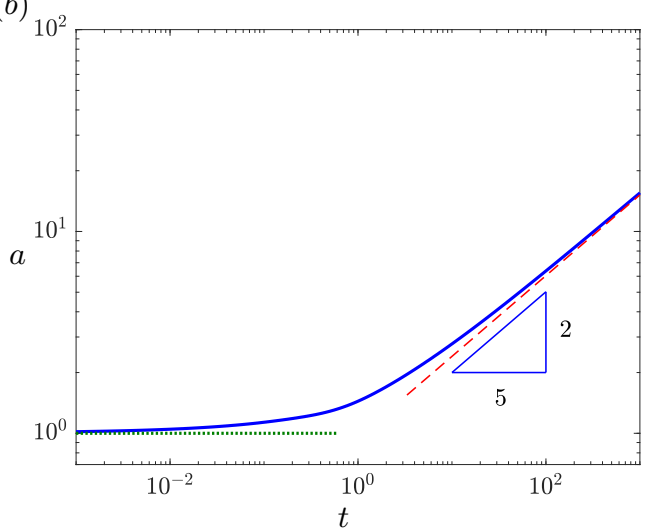

Figure 9: Log- $\log$ plots of the numerical solution to (6.4) with (2.24)-(2.26) (solid blue) with $q=2$ for $(a) h(0, t)$ and $(b) a(t)$, compared with the asymptotic predictions for early time, (6.8) (dotted green), and late time, (6.22)-(6.23) (dashed red). Here, $\delta=10^{-3}$.

of the apparent contact line for $\bar{t} \gg 1$

$$
\begin{aligned}
\bar{h}_{0}(0, \bar{t}) & \sim\left(\frac{9(q+4)}{8(4 q+7)}\right)^{1 / 3} C^{4 / 3} \bar{t}^{3 /(4 q+7)}, \\
a_{0}(\bar{t}) & \sim C \bar{t}^{(q+4) /(4 q+7)},
\end{aligned}
$$

where $C$ is defined in (6.18). We see that setting $q=0$ recovers (3.51) and (3.52). We note that we can obtain the exponents of these power-law dependencies using the scaling arguments, found at the end of $\S 3$. Also, as mentioned in $\S 3$, it is possible to use the Cox-Voinov law in the case of thickness-dependent injection to obtain the true late-time behaviour of the film spreading. In particular, our results (6.19) and (6.20) are valid for $1 \ll \bar{t}=\lambda_{q} t \ll \lambda_{q} / \delta^{(4 q+7) / 3}$ ( $c f$. comment immediately after (C 5) with $\left.q=0\right)$. We also remark that the aspect ratio for the problem is $\epsilon=h_{0} / a_{0} \sim t^{-(q+1) /(4 q+7)} \ll 1$ for large $t$ and $q>0$, and so, the thin-film hypothesis holds.

To illustrate our analysis for a particular value of $q$, we take $q=2$, for which $(6.15)$ simplifies substantially, and we can perform the integration in (6.16):

$$
\int_{0}^{1} \frac{1}{\left(a_{0}^{2}-x^{2}\right)^{2}} \mathrm{~d} x=\frac{\left(a_{0}^{2}-1\right) \operatorname{coth}^{-1}\left(a_{0}\right)-a_{0}}{2 a_{0}^{3}\left(a_{0}^{2}-1\right)} \sim \frac{1}{a_{0}^{4}} \quad \text { for } \quad a_{0} \gg 1 .
$$

We then have

$$
\begin{array}{r}
a_{0}(\bar{t}) \sim\left(\frac{50}{9}\right)^{1 / 5} \bar{t}^{2 / 5}, \\
\bar{h}_{0}(0, \bar{t}) \sim\left(\frac{5 \times 9^{1 / 3}}{4}\right)^{1 / 5} \bar{t}^{1 / 5} .
\end{array}
$$

In figure 9, we show $\log -\log$ plots of the maximum film thickness and the position of the apparent contact line for the case of thickness-inhibited injection with $q=2$. We see a very good agreement between the numerical solutions and the predicted asymptotic results for early time, given by (6.8), and late time, given by (6.22)-(6.23). 


\section{Conclusions and Further Work}

In this paper, we have considered the problem of surface-tension-driven flow of a viscous liquid along a flat surface with injection through part of the substrate. Throughout our work, we assumed that gravity could be neglected. We initially focused on the situation in which the injection rate was constant. We derived a fourth-order parabolic evolution equation for the thickness profile, along with appropriate boundary conditions, reflecting the injection in a fixed region. We assumed that a thin precursor layer was present initially along the surface, which resolved the stress singularity at the moving contact line. We used asymptotic analysis for early, $O(1)$, and late time in order to examine the behaviour of the solution, and obtained power-law dependencies on time for the maximum film thickness at the centre of the injection region, $h_{0}(0, t)$, and the position of the apparent contact line, $a_{0}(t)$. We recovered a version of Tanner's law at the apparent contact line, namely, $\partial h_{0} / \partial x \propto-\left(\dot{a}_{0}\right)^{1 / 3}$. For early time, we established a linear growth of the film thickness in the injection region and no movement of the contact line to leading order. We justified the absence of motion of the contact line to leading order for $O(1)$ time, while for late time, $t$, the maximum film thickness scales as $t^{3 / 7}$ and the position of the apparent contact line scales as $t^{4 / 7}$. Up to a multiplicative constant, the time-dependent aspect ratio is $h_{0}(0, t) / a_{0}(t) \sim t^{-1 / 7}$, as $t \rightarrow \infty$, which is consistent with the thinfilm assumption. We observed that the leading-order results for $h$ and $a$ depend on the precursor thickness, $\delta$. We also showed how to generalize classical results (such as the Cox-Voinov law) from the droplet-spreading problem in the case of injection to obtain the full late-time behaviour of the film.

Finally, we considered three generalizations to the problem: a power-law, point-source and thickness-dependent injection rate. In the first case, we looked at injection of the form $t^{k}$ with an exponent in the range $-1<k<1$, and established power-law behaviours for the maximum film thickness and the position of the apparent contact line as functions of time. We showed that, for late time, the film thickness increased for $k>-3 / 4$, tended to a constant for $k=-3 / 4$, and decreased for $k<-3 / 4$. For $k \geqslant 1$, the aspect ratio of the problem grows unboundedly with time and eventually invalidates the thin-film hypothesis. We also presented a simple inverse problem for determining the exponent of the power-law injection (assuming this exists) by observing the position of the apparent contact line, for example. In the point-source case, we obtained the same late-time behaviour as in the constant-injection-rate case. With regards to the thickness-dependent injection, we formulated the problem with injection of the form $1 / h^{q}$ for some exponent $q>0$. We obtained an integro-differential equation for the position of the apparent contact line for late time and simplified it to an ordinary differential equation. We found power-law behaviours for the maximum thickness of the film and the position of the apparent contact line, which were confirmed using scaling arguments. Unlike the time-dependent-injection case, we observed unbounded growth and spreading of the film for all $q>0$. We note that, as $q$ increases, the exponents in the spreading behaviour decrease, corresponding to a slowing down of the film growth and front spreading. Furthermore, we see a close relationship between the behaviour for $a(t)$ and $h(0, t)$ between the power-law-injection and the thickness-inhibited-injection cases; they are identical if $k=-3 q /(4 q+7)$. Interestingly, since $q>0, k \rightarrow-3 / 4$ as $q \rightarrow \infty$. This recovers the critical exponent for time-dependent injection at which the film thickness stops increasing for late time. This gives a relationship between the qualitative behaviours for late time of the maximum film thickness and the position of the apparent contact line with power-law-in-time and thickness-inhibited injection rates.

There are various key extensions to this work that we believe would be interesting. 
One would be to replace the flat substrate with a cylinder. This has the potential of introducing the well-known Rayleigh-Plateau instability, and a key question would be how the two principal curvatures affect the flow. Also of interest would be to study multiple fluid injection sites.

Our ultimate aim is to use the insight we have obtained by studying these surfacetension- and injection-driven flows to provide a better understanding of the operation of purification devices.

\section{Acknowledgements}

This publication is based on work partially supported by the EPSRC Centre For Doctoral Training in Industrially Focused Mathematical Modelling (EP/L015803/1) in collaboration with W. L. Gore and Associates. We would like to thank U. Beuscher and V. Venkateshwaran from Gore for their crucial contributions. IMG gratefully acknowledges support from the Royal Society through a University Research Fellowship. It is a pleasure to also thank D. Schwendeman, D. Vella and J. Oliver for invaluable discussions and comments. Finally, we are indebted to an anonymous referee for their insightful comments, which have significantly enhanced this paper.

\section{Appendix A. A Suitable Change of Coordinates for Numerical Solution}

When solving numerically (2.23) together with (2.24)-(2.26), we employ the following coordinate transformation

$$
\eta=\frac{x}{t^{1 / 4}}, \quad \tau=\log (t)
$$

In terms of $\eta$ and $\tau$, our problem for $h(x, t)=F(\eta, \tau)$ becomes

$$
\frac{\partial F}{\partial \tau}-\frac{1}{4} \eta \frac{\partial F}{\partial \eta}+\frac{1}{3} \frac{\partial}{\partial \eta}\left(F^{3} \frac{\partial^{3} F}{\partial \eta^{3}}\right)=\mathrm{e}^{\tau} \Theta\left(1-\mathrm{e}^{\tau / 4} \eta\right),
$$

subject to

$$
\begin{array}{rll}
\frac{\partial F}{\partial \eta}=\frac{\partial^{3} F}{\partial \eta^{3}}=0 & \text { at } & \eta=0, \\
F \rightarrow \delta & \text { as } & \eta \rightarrow \infty, \\
F=\delta & \text { at } & \tau \rightarrow-\infty .
\end{array}
$$

If we discretize this problem using an implicit linear Euler scheme and a sufficiently small time step, then our time domain for $\tau$ is much smaller than that for $t$ due to the exponential nature of the transformation in time. Thus, enlarging the $\tau$-domain marginally gives a much greater increase in the $t$-domain.

When solving the problem numerically, we require that the spatial step is bounded by the film height in order to ensure that the region near the capillary ripple is well resolved (Diez et al. 2001). Clearly, this presents computational issues as $\delta \rightarrow 0$. We employ the transformation

$$
F=\mathrm{e}^{G}
$$


and the problem for $G(\eta, \tau)$ becomes

$$
\begin{array}{r}
\frac{\partial G}{\partial \tau}-\frac{1}{4} \eta \frac{\partial G}{\partial \eta}+\frac{\mathrm{e}^{-G}}{3} \frac{\partial}{\partial \eta}\left(\mathrm { e } ^ { 4 G } \left(\left(\frac{\partial G}{\partial \eta}\right)^{3}+\right.\right. \\
\left.\left.+3 \frac{\partial G}{\partial \eta} \frac{\partial^{2} G}{\partial \eta^{2}}+\frac{\partial^{3} G}{\partial \eta^{3}}\right)\right) \\
=\mathrm{e}^{\tau-G} \Theta\left(1-\mathrm{e}^{\tau / 4} \eta\right),
\end{array}
$$

subject to

$$
\begin{aligned}
& \frac{\partial G}{\partial \eta}=\frac{\partial^{3} G}{\partial \eta^{3}}=0 \quad \text { at } \quad \eta=0, \\
& G \rightarrow \log (\delta)=\Upsilon \quad \text { as } \quad \eta \rightarrow \infty, \\
& G=\log (\delta)=\Upsilon \quad \text { at } \quad \tau \rightarrow-\infty .
\end{aligned}
$$

Since $|\log (\delta)|>\delta$, the requirement on the spatial step is less restrictive in the transformed version of the problem (A 7)-(A 10) than in the original formulation (A 2)-(A 5).

\section{Appendix B. Early-Time Asymptotics for the Power-Law Injection in the Limit $\delta \ll 1$}

We can make analytical progress and gain some insight into the behaviour of the film spreading in the boundary layer near $x=1$ by considering different temporal regimes. We begin by recalling the early-time result for the film thickness above the injection slot, (5.7), namely

$$
h(x, t)=\delta+\frac{t^{k+1}}{k+1} .
$$

For $t \ll \delta^{1 /(k+1)}$, we have $h \sim \delta$, and, thus, the inner solution satisfies the linear hyperdiffusion equation

$$
\frac{\partial h}{\partial t}+\frac{\delta^{3}}{3} \frac{\partial^{4} h}{\partial x^{4}}=t^{k} \Theta(1-x) .
$$

Scaling analysis suggests a spreading rate of the form $x-1 \sim\left(\delta^{3} t\right)^{1 / 4}$. Thus, we seek a solution of the form

$$
h \sim \delta+\frac{t^{k+1}}{k+1} \mathcal{H}(\xi)
$$

where $\xi=(1-x) /\left(\delta^{3} t\right)^{1 / 4}$, and $\mathcal{H}$ satisfies

$$
(k+1) \mathcal{H}-\frac{1}{4} \xi \frac{\partial \mathcal{H}}{\partial \xi}+\frac{1}{3} \frac{\partial^{4} \mathcal{H}}{\partial \xi^{4}}=(k+1) \Theta(\xi),
$$

with $\mathcal{H}(\xi) \rightarrow \Theta(\xi)$ as $\xi \rightarrow \pm \infty$.

When $t=O\left(\delta^{1 /(k+1)}\right)$, both terms in (B 1) are comparable in magnitude, and the non-linearity of $h^{3}$ in the original equation becomes important. For $t \gg \delta^{1 /(k+1)}$, we can consider both regions $x<1$ and $x>1$ separately. In $x<1, h \sim t^{k+1} /(k+1)$, and the spreading rate is $x-1 \sim\left(h^{3} t\right)^{1 / 4} \sim t^{(3 k+4) / 4} /(k+1)^{3 / 4}$. We, thus, seek a solution of the form

$$
h \sim \frac{t^{k+1}}{k+1} \mathcal{H}(\xi)
$$

where $\xi=(1-x) /\left(t^{3 k+4} /(k+1)^{3}\right)^{1 / 4}$, and $\mathcal{H}$ satisfies

$$
(k+1) \mathcal{H}-\frac{3 k+4}{4} \xi \frac{\partial \mathcal{H}}{\partial \xi}+\frac{1}{3} \frac{\partial}{\partial \xi}\left(\mathcal{H}^{3} \frac{\partial^{3} \mathcal{H}}{\partial \xi^{3}}\right)=(k+1) \Theta(\xi),
$$


with

$$
\begin{aligned}
\mathcal{H}(\xi) & \rightarrow 1 & \text { as } & \xi \rightarrow \infty, \\
\mathcal{H}=\mathcal{H}^{3}\left(\partial^{3} \mathcal{H} / \partial \xi^{3}\right) & =0 \quad \text { at } & \xi & =0 .
\end{aligned}
$$

The first condition ensures that the two non-decaying modes $\mathcal{H}-1 \propto 1$ and $\exp \left((3 / 4)(3(3 k+4) / 4)^{1 / 3} \xi^{4 / 3}\right)$ are excluded.

For $\xi \ll 1$ (i.e., near $x=1$ ), we balance the capillary and injection terms in (B 6). Assuming the solution is of the form $\mathcal{H} \propto \xi \log ^{p}\left(\xi^{r}\right)$, this yields

$$
\frac{\partial \mathcal{H}}{\partial \xi} \sim(12(k+1))^{1 / 4} \log ^{1 / 4}(1 / \xi)
$$

for which the first and second terms in (B 6) are indeed sub-dominant. Writing the position of the apparent contact line $a=1+b$, we expect to find a quasi-static region near $x=1$ with an apparent contact line at $x-1=b$. In this region, the capillary term dominates and the profile of the film is linear to leading order, $h \propto(1+b-x)$, with slope given by matching with (B9), resulting in

$$
\partial h / \partial x=-12^{1 / 4} t^{k / 4} \log ^{1 / 4}\left(t^{(3 k+4) / 4} /\left(b(k+1)^{3 / 4}\right)\right) .
$$

Using the Cox-Voinov law (see Appendix C), we obtain the spreading speed (and, thus, b) as

$$
\dot{b} \sim \frac{12^{3 / 4} t^{3 k / 4} \log ^{3 / 4}\left(t^{(3 k+4) / 4} /\left(b(k+1)^{3 / 4}\right)\right)}{9 \log \left(b t^{k / 4} / \delta\right)} .
$$

\section{Appendix C. Full Late-Time Asymptotics Using the Cox-Voinov Law}

In this appendix, we show how to obtain the full late-time behaviour of our model. Our starting point is the relationship between the apparent and microscopic contact angles $\theta_{o, i}$ given by the well-known Cox-Voinov law (Bonn et al. 2009)

$$
\theta_{o}^{3}-\theta_{i}^{3}=\frac{9 \mu \dot{a}}{\gamma} \log \left(L_{o} / L_{i}\right)
$$

where $L_{o, i}$ are the length scales of the outer and inner regions of the problem (in analogy to the terminology used in $\S 3)$. We use (3.32), (3.33) and (3.47)-(3.49) to find that $\log \left(L_{o} / L_{i}\right)=\log \left(a_{0} / \nu s\right) \sim \log \left(t / \delta a_{0}\right)=\log (h(0, t) / \delta)$ to leading order in $\delta$. Thus, with our non-dimensionalization and remembering that $\theta_{i}=0$ in our precursor-film regularization, we write $a_{0}=1+b$ to give

$$
\dot{b} \sim \frac{\theta_{o}^{3}}{9 \log \left(h_{0}(0, t) / \delta\right)} .
$$

For $1 \ll t \ll 1 / \lambda=\log ^{1 / 4}(1 / \delta)$, we linearize (3.53) and we find that

$$
b \sim \frac{3 t^{4}}{4 \log (1 / \delta)} \ll 1 .
$$

This can also be obtained from the Cox-Voinov law using (3.32) with $a_{0}=1, \theta_{o}=3 t$, and $h_{0}(0, t) \propto t$, and noting that $\log (t / \delta) \sim \log (1 / \delta)$ for the specified range of $t$. In the case where $t=O\left(\log ^{1 / 4}(1 / \delta)\right)$, we have $b=O(1)$, and the behaviour of the film spreading is given by our result (3.53). For the late-time regime, $t \gg \log ^{1 / 4}(1 / \delta), a_{0} \sim b \gg 1$, and, 
using $(\mathrm{C} 1)$, but this time with $\theta_{o}=3 t / a_{0}^{2}$ and $h_{0}(0, t) \propto t / a_{0}$ from (3.32), we obtain

$$
\dot{a}_{0} \sim \frac{3 t^{3}}{a_{0}^{6} \log \left(t / \delta a_{0}\right)} .
$$

Integrating the leading-order version of $(\mathrm{C} 4)$ in which we treat the logarithm as constant (which can be confirmed a posteriori), we find that $a_{0}$ scales as

$$
a_{0} \sim\left(\frac{21 t^{4}}{4 \log \left(t^{3 / 7} / \delta\right)}\right)^{1 / 7}
$$

We see that, for $t \gg \log ^{1 / 4}(1 / \delta), a_{0}(t)$ has a true power-law dependence on time, provided $t \ll 1 / \delta^{7 / 3}$.

In an analogous way, we can use the Cox-Voinov law in the case when the injection rate (and, therefore, volume) varies with time to obtain the full late-time behaviour. Again writing $a_{0}=1+b$ and repeating the above analysis, we find that, for $1 \ll t \ll$ $\log ^{1 /(3 k+4)}(1 / \delta), b \ll 1$ and is given by

$$
b \sim \frac{3 t^{3 k+4}}{(k+1)^{3}(3 k+4) \log (1 / \delta)},
$$

which is the linearization of (5.10). For $t=O\left(\log ^{1 /(3 k+4)}(1 / \delta)\right)$, we have $b=O(1)$, and the behaviour of the film spreading is given by our result (5.10).

For the late-time regime, $t \gg \log ^{1 /(3 k+4)}(1 / \delta), a_{0} \sim b \gg 1$, and we have

$$
a_{0} \sim\left(\frac{21 t^{3 k+4}}{(k+1)^{3}(3 k+4) \log \left(t^{(4 k+3) / 7} / \delta\right)}\right)^{1 / 7} .
$$

\section{REFERENCES}

AJAEv, V. S. 2012 Interfacial fluid mechanics: a mathematical modelling approach. Springer.

Anderson, D. M. \& Davis, H. 1995 The spreading of volatile liquid droplets on heated surfaces. Phys. Fluids 7 (2), 248-265.

Benilov, E. S., Chapman, S. J., Mcleod, J. B., Ockendon, J. R. \& Zubkov, V. S. 2010 On liquid films on an inclined plate. J. Fluid Mech. 663, 53-69.

Bonn, D., Eggers, J., Indekeu, J., Meunier, J. \& Rolley, E. 2009 Wetting and spreading. Rev. Mod. Phys. 81 (2), 739-805.

Carlson, A., Mandre, S. \& Mahadevan, L. 2015 Elastohydrodynamics of contact in adherent sheets , arXiv: 1508.06234.

Chilukuri, R., Aeling, D. \& Middleman, S. 1984 Removal of a thin liquid film from a flat surface using an axisymmetric impinging jet. J. Fluids Eng. 106 (2), 223-226.

Cox, R. G. 1986 The dynamics of the spreading of liquids on a solid surface. part 1. viscous flow. J. Fluid Mech. 168, 169-194.

Craster, R. V. \& Matar, O. K. 2009 Dynamics and stability of thin liquid films. Rev. Mod. Phys. 81, 1131-1198.

Davis, S. H. \& Hocking, L. M. 1999 Spreading and imbibition of viscous liquid on a porous base. Phys. Fluids 11 (1), 48-57.

Davis, S. H. \& Hocking, L. M. 2000 Spreading and imbibition of viscous liquid on a porous base. II. Phys. Fluids 12 (7), 1646-1655.

Diez, J. A., Kondic, L. \& Bertozzi, A. 2001 Global models for moving contact lines. Phys. Rev. E 63, 1-13.

Duffy, B. R. \& Moffatt, H. K. 1997 A similarity solution for viscous source flow on a vertical plane. Eur. J. Appl. Math. 8, 37-47.

Duffy, B. R. \& Wilson, S. K. 1997 A third-order differential equation arising in thin-film flows and relevant to tanner's law. Appl. Math. Lett. 10 (3), 63-68. 
Ehrhard, P. \& DAvis, S. H. 1991 Non-isothermal spreading of liquid drops on horizontal plates. J. Fluid Mech. 229, 365-388.

Fraysse, N. \& Homsy, G. M. 1994 An experimental study of rivulet instabilities in centrifugal spin coating of viscous Newtonian and non-Newtonian fluids. Phys. Fluids 6 (4), 14911504.

Guzman, R. \& Vasquez, D. A. 2016 Surface tension driven flow on a thin reaction front. Eur. Phys. J. Spec. Top. 225 (13-14), 2573-2580.

Halpern, D. \& Grotberg, J. B. 1992 Fluid-elastic instabilities of liquid-lined flexible tubes. J. Fluid Mech. 244, 615-632.

Hocking, G. C., Sweatman, W. L., Fitt, A. D. \& Breward, C. 2011 Deformations during jet-stripping in the galvanizing process. J. Engng Maths 70 (1-3), 297-306.

Hocking, L. M. 1980 Sliding and spreading of two-dimensional drops. Q. J. Mech. Appl. Math. $34(1), 37-55$.

Hocking, L. M. 1983 The spreading of a thin drop by gravity and capillarity. Q. J. Mech. Appl. Maths 36 (1), 55-69.

Hocking, L. M. 1992 Rival contact-angle models and the spreading of drops. J. Fluid Mech. 239, 671-681.

Howell, P. D. 2010 Surface-tension-driven flow on a moving curved surface. J. Engng Maths 45 (3), 283-308.

Howell, P. D., Robinson, J. \& Stone, H. A. 2013 Gravity-driven thin-film flow on a flexible substrate. J. Fluid Mech. 732, 190-213.

Howison, S. D., Moriarty, J. A., Ockendon, J. R., Terrill, E. L. \& Wilson, S. K. 1997 A mathematical model for drying paint layers. J. Engng Maths 32 (4), 377-394.

Huh, C. \& Scriven, L. E. 1971 Hydrodynamic model of steady movement of a solid/liquid/fluid contact line. J. Colloid Interface Sci. 35 (1), 85-101.

Huppert, H. E. 1982 The propagation of two-dimensional and axisymmetric viscous gravity currents over a rigid horizontal surface. J. Fluid Mech. 121, 43-58.

King, J. R. \& Bowen, M. 2001 Moving boundary problems and non-uniqueness for the thin film equation. Eur. J. Appl. Maths 12 (3), 321-356.

LACEY, A. A. 1982 The motion with slip of a thin viscous droplet over a solid surface. Stud. Appl. Maths 67 (3), 217-230.

Lister, J. R. 1992 Viscous flows down an inclined pane from point and line sources. J. Fluid Mech. 242, 631-653.

Lister, J. R., Peng, G. G. \& Neufeld, J. A. 2013 Viscous control of peeling an elastic sheet by bending and pulling. Phys. Rev. Lett. 111 (15), 1-5.

Mason, D. P. \& Momoniat, E. 2004 Axisymmetric spreading of a thin liquid drop with suction or blowing at the horizontal base. Intl J. Non-Linear Mech. 39, 1013-1026.

McEwan, A. D. \& TAYlor, G. I. 1966 The peeling of a flexible strip attached by a viscous adhesive. J. Fluid Mech. 26 (1), 1-15.

McKinley, I. S., Wilson, S. K. \& Duffy, B. R. 1999 Spin coating and air-jet blowing of thin viscous drops. Phys. Fluids $\mathbf{1 1}$ (1), 30-47.

Momoniat, E. \& Mason, D. P. 2007 Spreading of a thin film with suction or blowing including surface tension effects. Intl J. Comp. Maths Appl. 53 (2), 198-208.

Momoniat, E., Ravindran, R. \& Roy, S. 2010 The influence of slot injection/suction on the spreading of a thin film under gravity and surface tension. Acta Mech. 211 (1-2), 61-71.

Murphy, E. A. \& LeE, W. T. 2017 Mathematical modelling of contact lens moulding. IMA J. Appl. Maths 82 (3), 473-495.

Myers, T. G. 1998 Thin films with high surface tension. SIAM Rev. 40 (3), 441-462.

Oliver, J. M., Whiteley, J. P., Saxton, M. A., Vella, D., Zubkov, V. S. \& King, J. R. 2015 On contact-line dynamics with mass transfer. Eur. J. Appl. Maths 26 (5), $671-719$.

Oron, Alexander, Davis, Stephen H. \& Bankoff, S. George 1997 Long-scale evolution of thin liquid films. Rev. Mod. Phys. 69 (3), 931-980.

Savva, N. \& Kalliadasis, S. 2009 Two-dimensional droplet spreading over topographical substrates. Phys. Fluids 21 (9), 92102.

Savva, N. \& Kalliadasis, S. 2011 Dynamics of moving contact lines: A comparison between slip and precursor film models. EPL 94 (6). 
Savva, N. \& Kalliadasis, S. 2013 Droplet motion on inclined heterogeneous substrates. J. Fluid Mech. 725, 462-491.

Saxton, M. A., Vella, D., Whiteley, J. P. \& Oliver, J. M. 2017 Kinetic effects regularize the mass-flux singularity at the contact line of a thin evaporating drop. J. Engng Maths 106 (1), 47-73.

Saxton, M. A., Whiteley, J. P., Vella, D. \& Oliver, J. M. 2016 On thin evaporating drops: When is the d2-law valid? J. Fluid Mech. 792, 134-167.

Schwartz, L. W. \& Michaelides, L. E. 1988 Gravity flow of a viscous liquid down a slope with injection. Phys. Fluids 31 (1988), 397-399.

Sibley, D. N., Nold, A., Savva, N. \& Kalliadasis, S. 2015 A comparison of slip, disjoining pressure, and interface formation models for contact line motion through asymptotic analysis of thin two-dimensional droplet spreading. J. Engng Maths 94 (1), 19-41.

Smith, P. C. 1973 A similarity solution for slow viscous flow down an inclined plane. J. Fluid Mech. 58 (2), 275-288.

TAnner, L. H. 1979 The spreading of silicone oil drops on horizontal surfaces. J. Phys. D: Appl. Phys. 12, 1473-1484.

Thompson, A. B., Tseluiko, D. \& Papageorgiou, D. T. 2015 Falling liquid films with blowing and suction. J. Fluid Mech. 787, 292-330.

Tuck, E. O. \& Schwartz, L. W. 1990 A numerical and asymptotic study of some third-order ordinary differential equations relevant to draining and coating flows. SIAM Rev. 32 (3), $453-469$.

Voinov, O. V. 1976 Hydrodynamics of wetting. Fluid Dynamics 11 (5), 714-721.

Wilson, S. D. R. 1982 The drag-out problem in film coating theory. J. Engng Maths 16 (3), 209-221.

Wilson, S. K., Hunt, R. \& Duffy, B. R. 2000 The rate of spreading in spin coating. J. Fluid Mech. 413, 65-88.

Yatim, Y. M., Duffy, B. R. \& Wilson, S. K. 2012 Similarity solutions for unsteady shearstress-driven flow of newtonian and power-law fluids: Slender rivulets and dry patches. $J$. Engng Maths 73 (1), 53-69.

Yatim, Y. M., Duffy, B. R. \& Wilson, S. K. 2013 Travelling-wave similarity solutions for a steadily translating slender dry patch in a thin fluid film. Phys. Fluids 25 (5), 52103.

Yatim, Y. M., Wilson, S. K. \& Duffy, B. R. 2010 Unsteady gravity-driven slender rivulets of a power-law fluid. J. Nonnewton Fluid Mech. 165 (21-22), 1423-1430.

Zheng, Z., Fontelos, M. A., Shin, S., Dallaston, M. C., Tseluiko, D., Kalliadasis, S. \& Stone, H. A. 2018 Healing capillary films. J. Fluid Mech. 838, 404-434.

Zheng, Z., Griffiths, I. M. \& Stone, H. A. 2015 Propagation of a viscous thin film over an elastic membrane. J. Fluid Mech. 784, 443-464. 